\title{
Polymer
}

\section{Styrene and substituted styrene grafted functional polyolefins via nitroxide mediated polymerization}

\begin{tabular}{|r|l|}
\hline Journal: & Polymer Chemistry \\
\hline Manuscript ID & Draft \\
\hline Article Type: & Paper \\
\hline Date Submitted by the Author: & n/a \\
\hline Complete List of Authors: & $\begin{array}{l}\text { domenichelli, Ilaria; National Research Council, ICCOM - UoS di Pisa } \\
\text { Banerjee, Sanjib; National Institute for Interdisciplinary Science and } \\
\text { Technology CSIR, Materials Science and Technology Division; } \\
\text { Taddei, Sara; National Research Council, ICCOM - UoS di Pisa } \\
\text { Martinelli, Elisa; National Research Council, ICCOM - UoS di Pisa } \\
\text { PASSAGLIA, ELISA; National Research Council, ICCOM - UoS di Pisa; } \\
\text { Ameduri, Bruno; Ecole Nationale Sup rieure de Chimie (ENSC), } \\
\text { Laboratoire de Chimie Macromol culaire }\end{array}$ \\
\hline \multicolumn{2}{|l}{} \\
\hline
\end{tabular}

SCHOLARONE $^{\text {m }}$

Manuscripts 


\section{JUSTIFICATION}

The objectives of this study is the development of a strategy for using nitroxide functionalized polyolefin samples as macroinitiators for the synthesis of polyolefin- $g$-polystyrene graft copolymers using styrene or styrene derivatives as the second monomer.

Such a grafting occurs from bulk or surface-functionalized polyolefins (High density polyethylene and a poly(ethylene-co- $\alpha$-olefin) copolymer (EOC) modified with different functional 2,2,6,6-tetramethylpiperidine-1-oxyl radical (TEMPO) derivatives) via nitroxide mediated polymerization.

The "grafting from" polymerization of various styrene-based monomers led to polyolefin- $g$ poly styrene graft copolymers was evidenced by infrared (ATR-FTIR) and NMR (including solid state $\left.{ }^{19} \mathrm{~F}-\mathrm{NMR}\right)$ spectroscopies, size exclusion chromatography, thermogravimetric analysis, and differential scanning calorimetry.

The extent of grafting of PS chains onto the polyolefin backbone depended on the nature of macroinitiator, especially on the structure and cleavage temperature of alkoxy-amine derivatives created by TEMPO functionalization, on its content and on the adopted experimental conditions.

To the best of our knowledge, this is the first example of production of a library of polystyrene grafted polyolefins via a convenient nitroxide mediated strategy. 
Dear reviewers,

Would you please find attached the manuscript entitled "Styrene and substituted styrene grafted functional polyolefins via nitroxide mediated polymerization" by I. Domenichelli, S. Banerjee, S. Taddei, E. Martinelli, E. Passaglia (corresponding author) and B. Ameduri (corresponding author) for publication as an Article in Polymer Chemistry?

As you know, polyolefins are one of the most widely used plastic materials throughout the world. This arises a series of their unique features such as (a) low priced monomers and their easy availability, (b) the use of efficient and assessed catalytic systems. These features enable the preparation of a wide library of non-toxic and recyclable copolymers bearing a range of compositions and properties. Furthermore, the noteworthy chemical resistance and satisfactory mechanical properties of polyolefins guarantee their use in several other applications, such as food packaging, garbage disposal bags, ultra-high strength fibers for textile and building, and automobile bumpers. However, the presence of only sp3 hybridized carbons in the backbone and the resulting lack of functional groups limit the usefulness of polyolefin for applications which require satisfactory coating/adhesion features. The introduction of functionalities (even low amount of polar groups) can positively bring important properties such as toughness, adhesion, compatibility, barrier properties, surface properties (paintability, printability), solvent resistance, miscibility with other polymers, and rheological features without affecting their fundamental peculiarities.

The main objective of this study is the development of a strategy for using nitroxide functionalized polyolefin samples as macroinitiators for the synthesis of polyolefin- $g$-polystyrene graft copolymers using styrene or styrene derivatives as the second monomer. To the best of our knowledge, this is the first example of production of a library of polystyrene grafted polyolefins via a convenient nitroxide mediated strategy.

Several features have been highlighted in this manuscript:

i) The nitroxide mediated graft polymerization of styrene and/or substituted styrene derivatives from TEMPO functionalized polyolefins seems to be successful;

ii) ATR-FTIR, NMR, TGA, and DSC analyses enabled to fully highlight the grafting occurrence; in particular IR spectra of copolymers exhibited the frequencies attributed

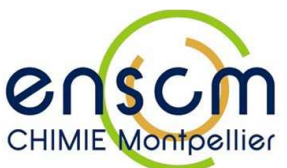

INGENIERIE ET ARCHITECTURES MACROMOLECULAIRES

8 Rue de l'Ecole Normale - 34296 MONTPELIIER

Tel : (33) (0) $4 \quad 67 \quad 144368$

Fax : (33) (0) $4 \quad 67 \quad 1472 \quad 20$

E-mail : bruno.ameduri@enscm.fr

website: www.iam.icgm.fr 
to aromatic moieties;

iii) The results evidenced that the grafting yield depended on the structure and stability of macro-alkoxyamine and on the experimental conditions with particular reference to reagents ratios owing to the lower temperature of the homolytic cleavage of bond generating the macroradical and the free nitroxides;

iv) These results pointed out that the functional polyolefin-alkoxyamines can be considered as "activated macromolecules" to be exploited as macroinitiators for the subsequent preparation of graft polymers with tuned/tailored structure-properties.

This work describes an original research on macromolecular science. It provides a detailed study of the reaction parameters for the synthesis of polystyrenegrafted polyolefins, which can be regarded as an important advance in polymer chemistry. Thus, we are confident that this manuscript will be of interest by the broad readership of Polymer Chemistry and hope that you will find it meets the high standard set by Polymer Chemistry for publication.

All co-authors have read the submitted manuscript and approved the final version. There is no conflict of interest. The manuscript has been submitted exclusively to Polymer Chemistry and has not been published or submitted simultaneously for publication elsewhere.

We are looking forward to receiving a positive decision regarding the manuscript.

Sincerely,

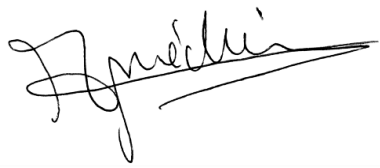

On behalf of the co-authors, B. AMEDURI

Directeur de Recherche au CNRS

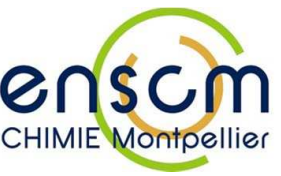

INGENIERIE ET ARCHITECTURES MACROMOLECULAIRES

8 Rue de l'Ecole Normale - 34296 MONTPELLIER

Tel : (33) (0) 467144368

Fax : (33) (0) $4671472 \quad 20$

E-mail : bruno.ameduri@enscm.fr

website: www.iam.icgm.fr 


\section{Styrene and substituted styrene grafted functional polyolefins via nitroxide mediated}

\section{polymerization}

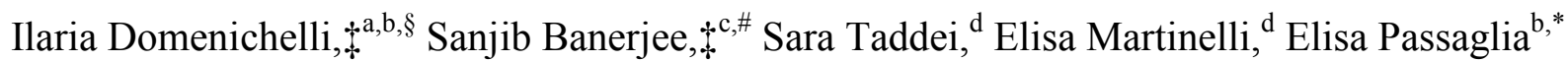
and Bruno Ameduri, ${ }^{\mathrm{c}, *}$

${ }^{a}$ Scuola Normale Superiore, Piazza dei Cavalieri 7, 56126 Pisa, Italy

${ }^{\mathrm{b}}$ CNR-ICCOM SS Pisa, Via Moruzzi 1, 56124 Pisa, Italy

${ }^{\mathrm{c}}$ Ingénierie et Architectures Macromoléculaires, Institut Charles Gerhardt, UMR 5253 CNRS, UM, ENSCM, Place Eugène Bataillon, 34095 Montpellier Cedex 5, France

${ }^{\mathrm{d}}$ Dipartimento di Chimica e Chimica Industriale, Via Moruzzi 13, 56124 Pisa, Italy

${ }^{\S}$ Present address: Department of Chemistry, Life Science and Environmental Sustainability, University of Parma, Parco Area delle Scienze 17/A, 43124 Parma, Italy

\#Present address: Material Science and Technology Division, CSIR-National Institute for Interdisciplinary Science and Technology, Thiruvananthapuram 695019, Kerala, India †These authors contributed equally to this manuscript.

Abstract: The grafting of (functionalized) polystyrene from bulk or surface-functionalized polyolefins via nitroxide mediated polymerization is described. High density polyethylene and a poly(ethylene-co- $\alpha$-olefin) copolymer (EOC) modified with different functional 2,2,6,6tetramethylpiperidine-1-oxyl radical (TEMPO) derivatives were used as macroinitiators for the "grafting from" polymerization of various styrene-based monomers to offer polyolefin-g-poly styrene graft copolymers. The successful grafting of styrene and styrene derivatives was demonstrated by complementary analyses such as infrared (ATR-FTIR) and NMR spectroscopies, size exclusion chromatography, thermogravimetric analysis, and differential 
scanning calorimetry. Typically, IR of the grafted copolymers showed the bands attributable to the aromatic moieties and the obtained thermograms evidenced a lower degradation temperature for the grafted copolymers compared to that of polyolefin starting materials. In addition, solid state ${ }^{19} \mathrm{~F}-\mathrm{NMR}$ was chosen to confirm the growth of polystyrene (PS) chains when EOC functionalized with a fluoroalkyl TEMPO was used for the NMP. The extent of grafting of PS chains onto the polyolefin backbone was found to depend on the nature of macroinitiator, especially on the structure and cleavage temperature of alkoxy-amine derivatives created by TEMPO functionalization, on its content and on the adopted experimental conditions.

\section{Introduction}

Polyolefins are the class of plastic materials most widespread throughout the world owing to a series of their unique features such as low priced monomers and their easy availability, the use of efficient and assessed catalytic systems. These features allow the preparation of copolymers with a broad range of compositions and properties by cheap and environmentally friendly productive processes providing non-toxic and recyclable polymers. Furthermore, the noteworthy chemical resistance and satisfactory mechanical properties of polyolefins guarantee their use in several kinds of applications, as food packaging, garbage disposal bags, ultra-high strength fibers for textile and building and automobile bumpers. However, the presence of only $\mathrm{sp}^{3}$ hybridized carbons in the backbone and the resulting lack of functional groups do not allow the polyolefin to be useful for applications which require good coating/adhesion characteristics. ${ }^{1}$ The insertion of functionalities (even low amount of polar groups) can positively bring important properties such as toughness, adhesion, compatibility, barrier properties, surface properties (paintability, printability), solvent resistance, miscibility with other polymers, and rheological features ${ }^{2}$ 
without affecting their fundamental peculiarities. Among the various modification techniques, the "grafting from" copolymerization approach is quite successful, because it allows the synthesis of well-structured materials with fascinating behaviour. ${ }^{3}$ The most used technique to obtain graft copolymer from the polyolefins is certainly the direct grafting of vinyl monomers onto the polymer backbone by means of radical initiators. ${ }^{4-6}$ Nevertheless, this methodology suffers from the lack of control over the molar mass and the occurrence of side reactions considerably affecting the final macromolecular architecture. ${ }^{7}$ However, the development of several reversible deactivation radical polymerization (RDRP) reactions, among which the oldest one is represented by the nitroxide mediated polymerization (NMP) is a useful tool for designing the synthesis of graft copolymers. ${ }^{8,9}$ After the Georges et al.'s first experiment, ${ }^{8}$ aimed at using 2,2,6,6-tetramethylpiperidine-1-oxyl radical (TEMPO) as controlling agent in the styrene polymerization, significant developments on the NMP technique were achieved, largely by demonstrating the ability of TEMPO to control the polymerization of styrene or styrene derivatives. $^{7,}$ 10-16 Usually, the standard procedure to obtain grafted copolymers via the NMP technique is as follows: firstly, the functionalization of the polymer with nitroxides and subsequently the growth of polymer brushes from the alkoxyamine sites by heating such a macroalkoxyamine in the presence of a monomer. In general, the synthesis of macroalkoxyamines as unimolecular systems by employing polyolefins as a matrix, requires a multi-step process without complete control of side reactions. ${ }^{17-19} \mathrm{~A}$ feasible alternative to these techniques is represented by the nitroxide radical coupling (NRC) reaction. NRC reaction has emerged as an excellent tool to add specific functionalities to different polymers, by a one-step procedure and by using ad hoc synthesized nitroxides (bearing specific functionalities) which are able to quickly react with the macroradicals formed by $\mathrm{H}$-abstraction owing to radical initiator. ${ }^{20}$, 
${ }^{21}$ Furthermore, this process shows a great compatibility with different functional groups and by tuning the nitroxide/initiator ratio, it is possible to achieve satisfactory grafting degrees by keeping under control the macromolecular architecture, although the functionalization is achieved in the melt by radicals.

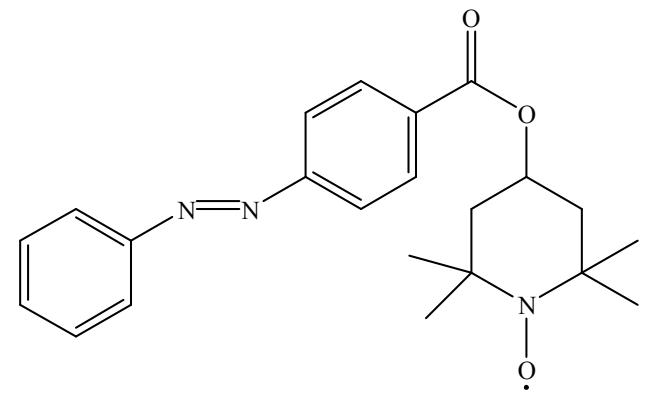

AzO-TEMPO

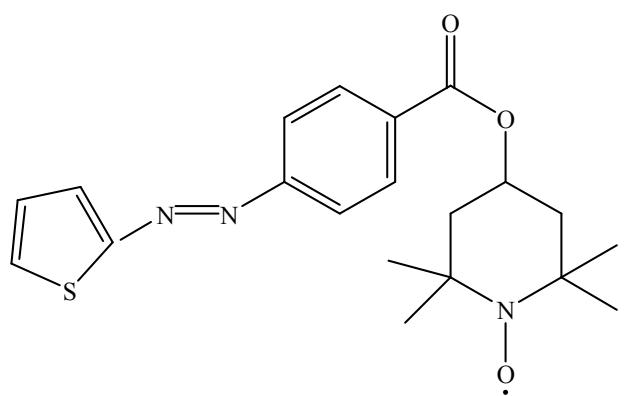

THiO-TEMPO

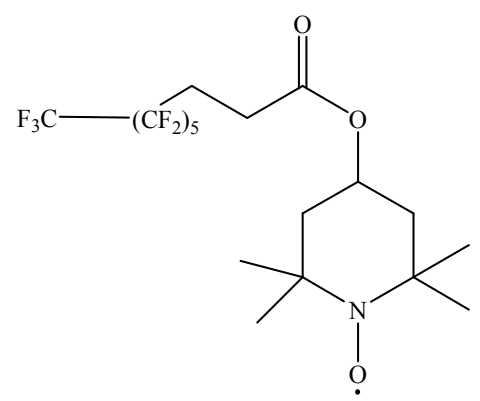

FluO-TEMPO

Figure 1 Structure of 4-(phenylazo)-benzoyloxy-2,2,6,6-tetramethylpiperidine-1-oxyl radical (AzO-TEMPO), 4-(2-thienylazo)-benzoyloxy-2,2,6,6-tetramethylpiperidine-1-oxyl (THiOTEMPO), and 4-(4,4,5,5,6,6,7,7,8,8,9,9,9-tridecafluorononanoyloxy)-2,2,6,6tetramethylpiperidine-1-oxyl (FluO-TEMPO).

On the basis of this literature scenario, in this work, polyolefin samples, previously functionalized with different TEMPO derivatives by NRC reaction, were used as macroinitiators for the synthesis of polyolefin-g-polystyrene graft copolymers using styrene or styrene derivatives as the second monomer. Indeed, polystyrene chains (PS) can grow up from the polyolefin backbone, by taking advantage of the presence of TEMPO, in agreement with 
literature on the "grafting from" TEMPO functionalized polymers/surfaces. ${ }^{17-19,22,23}$ On the other hand, while Takahara's group ${ }^{24}$ studied the controlled insertion of styrene into the TEMPO- based polyurethane backbone to yield segmented copolymers dispersing urethane groups homogeneously distributed in the main chain, Sundholm et al. ${ }^{25-28}$ reported styrene and sulfonated styrene grafted from poly(vinylidene fluoride) after quenching the radicals by a nitroxide. The graft copolymers were obtained in heterogeneous medium and the resulting graft copolymers displayed interesting proton conducting membranes.

The polyolefins used as backbone for growing the PS chains are functionalized with the nitroxides shown in Fig. 1. ${ }^{29,30}$ In particular, high density polyethylene (HDPE) was reacted with 4-(phenylazo)-benzoyloxy-2,2,6,6-tetramethylpiperidine-1-oxyl radical (AzO-TEMPO) and 4(2-thienylazo)-benzoyloxy-2,2,6,6-tetramethylpiperidine-1-oxyl radical (THiO-TEMPO). Also, a poly(ethylene-co-olefin) copolymer (EOC) was grafted with AzO-TEMPO and 4(4,4,5,5,6,6,7,7,8,8,9,9,9-tridecafluorononanoyloxy)-2,2,6,6-tetramethylpiperidine-1-oxyl (FluOTEMPO) in the bulk ${ }^{27,28}$ or through photograftingtechnique ${ }^{30}$.

All these samples, functionalized with the above mentioned nitroxides were used as macroinitiators to polymerize styrene derivatives in solution or in bulk and, after purification, the products were characterized by ATR-FTIR and NMR spectroscopies, size exclusion chromatography (SEC), and thermal analysis (TGA, DSC) to evidence the grafting of PS brushes.

\section{Experimental Section}

\section{Materials}


The random poly(ethylene-co-olefin) copolymer containing $38 \mathrm{wt} \%$ of 1-octene comonomer $(13.3 \mathrm{~mol} \%)\left(\right.$ EOC) and having $M_{\mathrm{n}}=50000 \mathrm{~g} / \mathrm{mol}, M_{\mathrm{w}}=102000 \mathrm{~g} / \mathrm{mol}$, dispersity $=2.04$, density $=0.87 \mathrm{~g} / \mathrm{cm}^{3}$ and melt flow rate $(\mathrm{MFR})=4.7 \mathrm{~g} / 10\left(2.16 \mathrm{~kg}\right.$ at $\left.190^{\circ} \mathrm{C}\right) \mathrm{min}$, and high density polyethylene (HDPE) Lacqtene ${ }^{\circledR}$ 2070MN60 produced by Arkema having $M_{\mathrm{n}}=21000$ $\mathrm{g} / \mathrm{mol}, M_{\mathrm{w}}=86000 \mathrm{~g} / \mathrm{mol}$, dispersity $=4.10$,density $=0.96 \mathrm{~g} / \mathrm{cm}^{3}$ and MFR $=7 \mathrm{~g} / 10 \mathrm{~min}(2.16$ $\mathrm{Kg}$ at $190^{\circ} \mathrm{C}$ ) were used as polymer matrix. Styrene (S) (Fluka) was washed with $5 \% \mathrm{NaOH}$ and water. After drying over $\mathrm{Na}_{2} \mathrm{SO}_{4}$, it was distilled under reduced pressure. Styrene (S) (Sigma Aldrich) pentafluorostyrene $\left(\mathrm{F}_{5} \mathrm{~S}\right)$ (Sigma Aldrich), 4-sodium styrenesulfonate (SS) (Sigma Aldrich), vinylbenzylchloride (VBC, Sigma Aldrich), were used after passing them through alumina column to remove the inhibitor. 1,2,4-Trichlorobenzene (Sigma Aldrich) was used without purification. The syntheses of HDPE- $g$-THiO-TEMPO, HDPE- $g$-AzO-TEMPO, EOC-gAzO-TEMPO, EOC-g-FluO-TEMPO and EOC-g-FluO-TEMPO-S were carried out as reported previously. ${ }^{29-30}$ Azobisisobutyronitrile (AIBN) was purchased from Aldrich.

Radical copolymerization of styrene and styrene derivatives onto the PE-g-X-TEMPO from the Nitroxide Mediated Polymerization (NMP) technique

Method 1: This method was applied to polymerize S from HDPE-g-AzO-TEMPO, HDPE-gTHiO-TEMPO and EOC-g-AzO-TEMPO. The copolymerization was carried out in a $50 \mathrm{~mL}$ flask where the macroinitiator $(500 \mathrm{mg})$ was dissolved in $10 \mathrm{~mL}$ of 1,2,4-trichlorobenzene at 120 ${ }^{\circ} \mathrm{C}$. Separately, $\mathrm{S}$ was degassed by $\mathrm{N}_{2}$ bubbling for $30 \mathrm{~min}$. The monomer was then transferred into the solution of functionalized polyethylene. The reaction was stopped after $48 \mathrm{~h}$ and the crude product was washed by extraction via centrifugation in chloroform or acetone for HDPE and EOC samples, respectively. The recovered solid was then dried under vacuum at $80{ }^{\circ} \mathrm{C}$ overnight. The same method was also used to polymerize $\mathrm{S}$ and its derivatives such as $\mathrm{F}_{5} \mathrm{~S}, \mathrm{SS}$ 
and VBC from EOC-g-AzO-TEMPO. The used reagents ratios and quantities are listed in Table 1. The products were characterized by Total Attenuated Reflection-Fourier Transform Infrared spectroscopy (ATR-FTIR) and Thermogravimetric analysis (TGA).

Method 2: a) The TEMPO mediated radical copolymerization from surface-functionalized polyolefin EOC-g-FluO-TEMPO-S (as film of about $300 \mathrm{mg}$ and $6 \mathrm{~cm} \times 6 \mathrm{~cm}$ ) with $\mathrm{S}$ was performed in a $50 \mathrm{~mL}$ flask where the macroinitiator was immersed in $5 \mathrm{~mL}$ of the monomer. After degassing by $\mathrm{N}_{2}$ bubbling for $30 \mathrm{~min}$, the sample was heated, under stirring, at $120{ }^{\circ} \mathrm{C}$ for $72 \mathrm{~h}$. Then, the crude product was washed by extraction in chloroform in order to separate the polyethylene from the free PS. Then, the film was recovered and dried under vacuum at $80{ }^{\circ} \mathrm{C}$ overnight and characterized by ATR-FTIR, TGA and Differential Scanning Calorimetry (DSC). b) The bulk-functionalized polyolefin EOC-g-FluO-TEMPO (400 mg) and $\mathrm{S}(10 \mathrm{~mL})$ were introduced under nitrogen in a dry Schlenk and left under stirring at room temperature for $1 \mathrm{~h}$. Then, the mixture was degassed by four freeze-pump-thaw cycles and the polymerization was left to proceed at $130{ }^{\circ} \mathrm{C}$ for $2.7 \mathrm{~h}$. The crude product was purified by repeated precipitations from methanol and the filtered solid was washed by extractions in THF and hot acetone in order to remove the unreacted polyolefin and PS, respectively. The recovered solid was finally dried under vacuum until constant weight and characterized by ATR-FTIR, TGA, DSC and size exclusion chromatography (SEC) analysis $\left(M_{\mathrm{n}}=165000 \mathrm{~g} / \mathrm{mol}\right.$ and $\left.M_{\mathrm{w}} / M_{\mathrm{n}}=1.46\right)$.

For a comparison purpose, a PS sample was prepared by using radical polymerization approach without the polyolefin substrate. In a 50 mL-flask, $3 \mathrm{~mL}$ of $\mathrm{S}$, previously degassed by $\mathrm{N}_{2}$ bubbling for $30 \mathrm{~min}$, and $43 \mathrm{mg}$ of AIBN were added. The reaction was left to proceed at $70{ }^{\circ} \mathrm{C}$ for $6 \mathrm{~h}$. The crude product was then purified by repeated (2) precipitations from methanol. The product was analyzed by SEC $\left(M_{\mathrm{n}}=42000 \mathrm{~g} / \mathrm{mol}\right.$ and $\left.M_{\mathrm{w}} / M_{\mathrm{n}}=3.56\right)$, TGA and DSC $(\mathrm{Tg}=$ 
$\left.99.6^{\circ} \mathrm{C}\right)$.

\section{Characterization}

Total Attenuated Reflection-Fourier Transform Infrared spectroscopy (ATR-FTIR)

IR spectra of the polymers were recorded using a PerkinElmer Spectrum ATR mode (diamond), from 4500 to $400 \mathrm{~cm}^{-1}$ as analysis range with an accuracy of $\pm 2 \mathrm{~cm}^{-1}$ and 16 scans.

\section{Thermogravimetric analysis (TGA)}

TGA was carried out by using the Seiko EXSTAR 7200 TGA/DTA instrument. In a typical experiment, the sample (about $10 \mathrm{mg}$ ) was placed in an alumina pan and the heating was achieved at a standard rate of $10^{\circ} \mathrm{C} / \mathrm{min}$ from 30 to $600{ }^{\circ} \mathrm{C}$ under nitrogen flow.

\section{Differential Scanning Calorimetry (DSC)}

DSC analysis was carried out using a Perkin-Elmer DSC-4000 differential scanning calorimeter thermal analyzer equipped with a 3 stage cooler able to reach $-130{ }^{\circ} \mathrm{C}$. Thermal scans were conducted on 10-15 mg samples under nitrogen atmosphere. Prior to the analyses, the instrument was calibrated by using indium (m.p. $156.6{ }^{\circ} \mathrm{C}, \Delta \mathrm{H}=28.5 \mathrm{~J} / \mathrm{g}$ ) and zinc (m.p. $419.5{ }^{\circ} \mathrm{C}$ ). $\mathrm{HDPE}$ samples were heated from 30 to $180^{\circ} \mathrm{C}$ then cooled to $30^{\circ} \mathrm{C}$ and heated again to $180^{\circ} \mathrm{C}$ at a cooling/heating rate of $10^{\circ} \mathrm{C} / \mathrm{min}$. EOC samples were cooled to $-90^{\circ} \mathrm{C}$, heated to $90^{\circ} \mathrm{C}$, cooled to $-90^{\circ} \mathrm{C}$ and heated one more time to $120^{\circ} \mathrm{C}$ at a cooling/heating rate of $10^{\circ} \mathrm{C} / \mathrm{min}$. PS was heated from 0 to $140^{\circ} \mathrm{C}$ then cooled to $0^{\circ} \mathrm{C}$ and heated again to $140^{\circ} \mathrm{C}$ at a cooling/heating rate of 10 ${ }^{\circ} \mathrm{C} / \mathrm{min}$.

\section{Size Exclusion Chromatography (SEC)}

SEC analysis of sample (EOC-g-FluO-TEMPO)_PS was carried out with a Jasco PU-2089 chromatograph equipped with two PL-gel mixed D 2065-CO thermostat columns, a Jasco 2031- 
RI plus refracting index detector and a 2077-UV plus UV detector. Polystyrene standards were used for calibration. Chloroform was the eluent with a flow rate of $1 \mathrm{~mL} / \mathrm{min}$. SEC analysis of sample PS was performed with Agilent Technologies 1200 Series instrument equipped with an Agilent degasser, an isocratic HPLC pump, an Agilent refractive index (RI) detector, and two PLgel $5 \mu \mathrm{m}$ MiniMIX-D columns conditioned at $35^{\circ} \mathrm{C}$. Chloroform $\left(\mathrm{CHCl}_{3}\right)$ was used as the mobile phase at a flow rate of $0.3 \mathrm{~mL}$ min-1. The system was calibrated with polystyrene standards in a range from 500 to $3 \times 105 \mathrm{~g}$ mol-1. Sample was dissolved in $\mathrm{CHCl}_{3}(2 \mathrm{mg} \mathrm{mL}-1)$ and filtered through a 0.20 micron syringe filter before analysis. Mn and $\mathrm{Mw}$ were determined using Agilent ChemStation software.

\section{Solution Nuclear Magnetic Resonance (NMR) spectroscopy}

${ }^{1} \mathrm{H}-\mathrm{NMR}$ spectra were recorded at room temperature on a Bruker Advance DRX 400 spectrometer. Samples were prepared in $\mathrm{CDCl}_{3}$ by dissolving $25-30 \mathrm{mg}$ of product in $0.8 \mathrm{ml}$ of solvent.Coupling constants and chemical shifts are given in hertz $(\mathrm{Hz})$ and parts per million(ppm), respectively. The instrumental parameters for recording ${ }^{1} \mathrm{H}$ NMR spectra were as follows: flip angle $90^{\circ}$, acquisition time $4.5 \mathrm{~s}$, pulse delay $2 \mathrm{~s}$, number of scans 32 .

\section{Solid State Nuclear Magnetic Resonance (NMR) spectroscopy}

Solid state NMR was used to qualitatively characterize the surface grafting. The NMR spectra were recorded on a 1 Hone pulse (OP) Varian VNMRS instrument $(400 \mathrm{MHz})$ at $25{ }^{\circ} \mathrm{C}$, using a $3.2 \mathrm{~mm}$-diameter rotor.

\section{Results and discussions}

Synthesis of polystyrene grafted polyethylene via the "grafting from" technique

Depending on the technique used to obtain the macroinitiators (bulk functionalization or surface 
grafting) and on the basis of their collected functionalization degree values (FD $\mathrm{X}$-TEMPO), two different methodologies were adopted to grow polymer chains (Scheme 1): First, HDPE-g-AzOTEMPO, HDPE-g-THiO-TEMPO and EOC-g-AzO-TEMPO ${ }^{31}$ were dissolved in 1,3,5trichlorobenzene and then mixed with S monomer at $120^{\circ} \mathrm{C}$ for $48 \mathrm{hr}$ (Method 1). EOC- $g$-FluOTEMPO and EOC-g-FluO-TEMPO-S ${ }^{30}$ were mixed with $\mathrm{S}$ monomer (liquid) and the embedded particles/films were heated up to $130 / 120^{\circ} \mathrm{C}$ for $2.7 / 72 \mathrm{~h}$ (Methods $2 \mathrm{a}$ and $\mathrm{b}$, respectively). In addition, the EOC-g-AzO-TEMPO was chosen as the matrix to graft, according to method 1, copolymer brushes constituted by styrene and some styrene derivatives such as pentafluorostyrene $\left(\mathrm{F}_{5} \mathrm{~S}\right)$, 4-vinylbenzylchloride (VBC) and sodium 4-styrene sulfonate (SS) (Table 1). After polymerization, all the samples were washed with chloroform or acetone to eliminate the ungrafted PS.
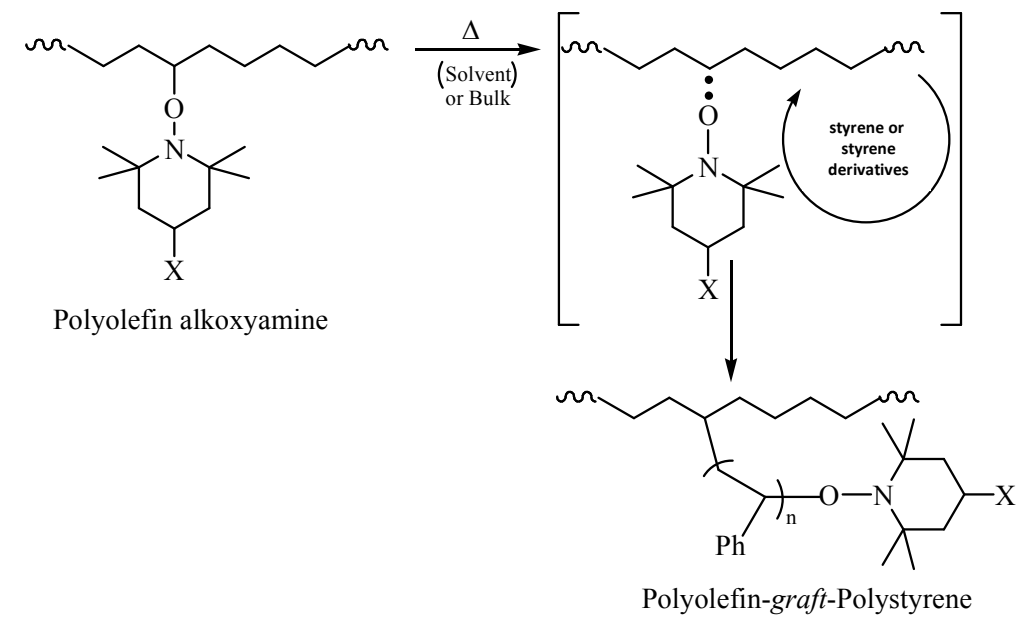

method 1: Solvent=1,2,4-trichlorobenzene; $\mathrm{X}=\mathrm{AzO}$; THiO; polyolefin: EOC; HDPE method 2: Bulk; $X=F l u O ;$ polyolefin: EOC

Scheme 1. Simplified grafting reaction by using methods 1 and 2 (see Figure 1 and Table 1 for details)

To evidence the PS grafting, the ATR-FTIR spectra of the macroalkoxyamines, before and after polymerization runs, were compared. All the spectra were normalized with respect to the band at $720 \mathrm{~cm}^{-1}$ assigned to the $\mathrm{CH}_{2}$ rocking absorption of polyethylene. Concerning the 
products obtained from method 1, no bands ascribable to the grafting of comonomers were observed with the exception of products labeled (EOC-g-AzO-TEMPO)_PS and (EOC-g-AzOTEMPO)_poly(S-co-SS) (Figs. S1-S2 and Fig. 2). Indeed, the IR spectrum of (EOC-g-AzOTEMPO)_PS sample showed the presence of a band centered at $699 \mathrm{~cm}^{-1}$ which may be attributed to the out-of-plane bending of the $\mathrm{CH}$ groups of the polystyrene aromatic ring (Fig. S2).

Table 1 Experimental conditions of the radical (co)polymerization of functionalized polyolefins with $\mathrm{S}$ and styrene derivatives ${ }^{\S}$.

\begin{tabular}{|c|c|c|c|c|c|c|}
\hline Run & Sample & $\begin{array}{c}\text { monomer } \\
\text { A }\end{array}$ & $\begin{array}{c}\text { monomer } \\
\text { B }\end{array}$ & $\begin{array}{c}\text { FD }_{\text {XTEMPO }} \\
\% \mathrm{~mol}\end{array}$ & $\begin{array}{c}\mathrm{T} \\
\left({ }^{\circ} \mathrm{C}\right) \\
\end{array}$ & $\begin{array}{r}\text { time } \\
\text { (h) }\end{array}$ \\
\hline 1 & (EOC-g-AzO-TEMPO)_PS ${ }^{\mathrm{a}}$ & $\mathrm{S}$ & - & 0.18 & 120 & 48 \\
\hline 2 & (HDPE-g-AzO-TEMPO)_PS ${ }^{\mathrm{a}}$ & S & - & 0.12 & 120 & 48 \\
\hline 3 & (HDPE-g-THiO-TEMPO)_PS ${ }^{\mathrm{a}}$ & S & - & 0.11 & 120 & 48 \\
\hline 4 & $\left(\right.$ EOC-g-AzO-TEMPO)_poly $\left(\mathrm{S}-c o-\mathrm{F}_{5} \mathrm{~S}\right)^{\mathrm{b}}$ & $\mathrm{S}$ & $\mathrm{F}_{5} \mathrm{~S}$ & 0.18 & 120 & 48 \\
\hline 5 & $(\text { EOC-g-AzO-TEMPO)_poly(S-co-SS })^{\mathrm{b}}$ & S & SS & 0.18 & 120 & 48 \\
\hline 6 & $(\text { EOC-g-AzO-TEMPO)_poly(S-co-VBC })^{\mathrm{b}}$ & $\mathrm{S}$ & VBC & 0.18 & 120 & 48 \\
\hline 7 & (EOC-g-FluO-TEMPO-S)_PS ${ }^{\mathrm{c}}$ & $\mathrm{S}$ & - & 0.06 & 120 & 72 \\
\hline 8 & (EOC-g-FluO-TEMPO)_PS ${ }^{\mathrm{c}}$ & $\mathrm{S}$ & - & 0.12 & 130 & 2.7 \\
\hline 9 & (EOC)_PS & $\mathrm{S}$ & - & - & 120 & 72 \\
\hline
\end{tabular}

${ }^{\S}$ Functionalized polymers: EOC- $g$-AzO-TEMPO; HDPE-g-AzO-TEMPO; HDPE- $g$-THiO-TEMPO prepared as reported in ref 27 and 28; EOC-g-FluO-TEMPO and EOC-g-FluO-TEMPO-S prepared as reported in ref 29; $\mathrm{FD}_{\mathrm{XTEMPO}}(\% \mathrm{~mol})$ is the number of X-TEMPO molecules grafted per 100 monomer units and corresponds to the theoretical number of reactive sites of the macroinitiators. $\mathrm{S}, \mathrm{F}_{5} \mathrm{~S}, \mathrm{SS}$ and $\mathrm{VBC}$ stand for styrene, pentafluorostyrene, 4-sodium styrene sulfonate, and 4-vinylbenzylchloride, respectively

a: Conditions method 1: Solvent used $=1,2,4$-trichlorobenzene $10 \mathrm{~mL} ;\left([\mathrm{S}]_{0} /[\mathrm{X}-\mathrm{TEMPO}]_{0}=180\right.$;

b: Conditions method 1: Solvent used $=1,2,4$-trichlorobenzene $10 \mathrm{~mL}$; $\left([\mathrm{S}+\mathrm{S} \text { derivatives }]_{0} /[\mathrm{X}-\mathrm{TEMPO}]_{0}=360\right.$; $[\mathrm{S}] /[\mathrm{S}$ derivatives $]=1 / 1$

: Conditions method 2: Bulk reaction; $\left([\mathrm{S}]_{0} /[\text { FluO-TEMPO }]_{0}=6900\right.$ for $2 \mathrm{a} ;[\mathrm{S}]_{0} /[\text { FluO-TEMPO }]_{0}=7100$ for $2 \mathrm{~b}$

Furthermore, the ATR-FTIR spectrum of (EOC-g-AzO-TEMPO)_poly(S-co-SS) graft copolymer displays a band of low intensity at ca. $1040 \mathrm{~cm}^{-1}$ that may be related to $-\mathrm{SO}_{3}$ group 
symmetric vibration, ${ }^{32,33}$ suggesting the grafting of S and SS although in low yields (Fig. 2).

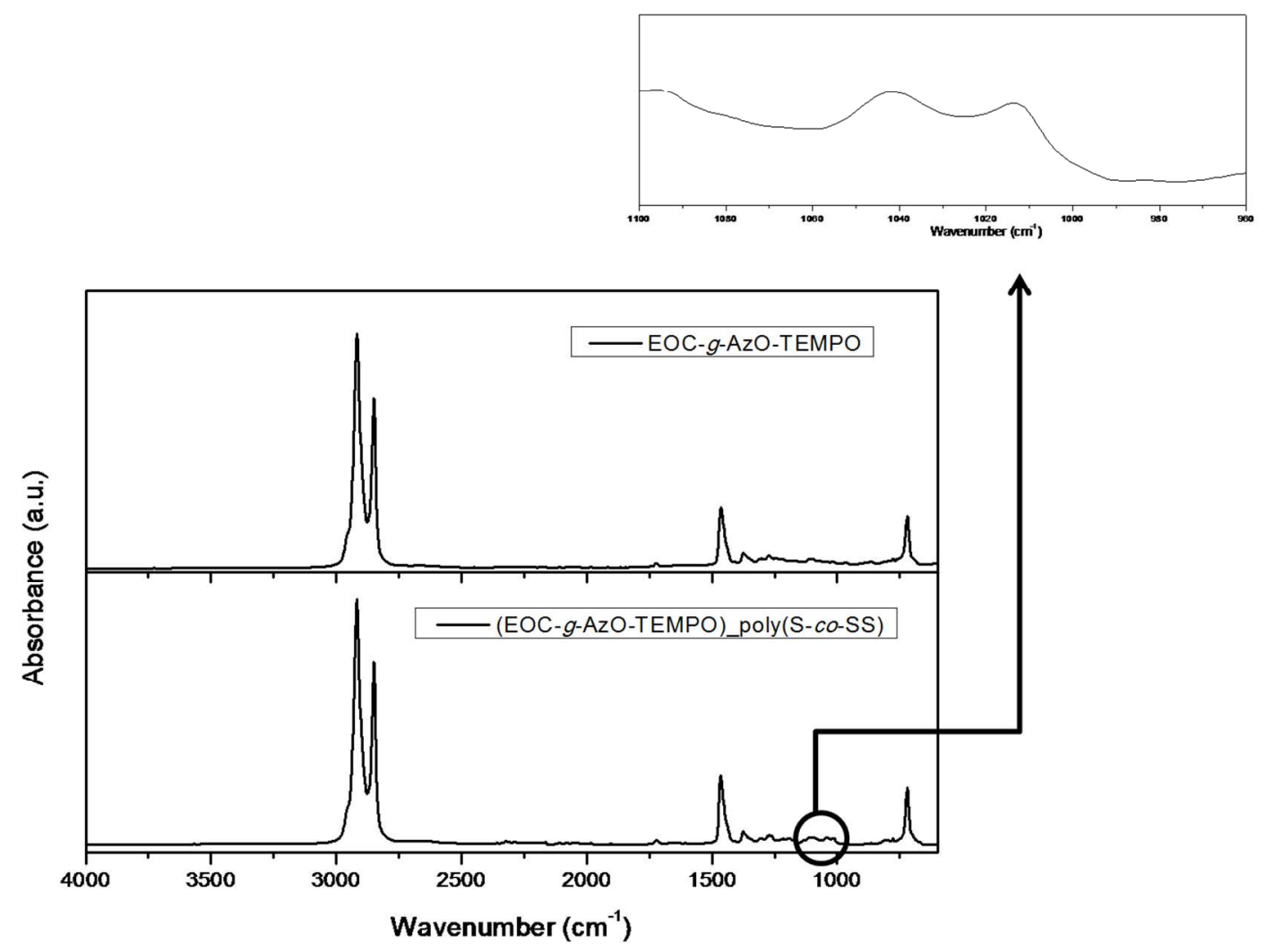

Figure 2 ATR-FTIR spectra of the EOC-g-AzO-TEMPO (upper spectrum) and (EOC-g-AzOTEMPO)_poly(S-co-SS) (lower spectrum, run 5, Table 1).

Therefore, on the basis of these preliminary evidences, it seems clear that, by using method 1 and macroinitiators derived from possible cleavage of grafted AzO-TEMPO and THiO-TEMPO, the grafting of $\mathrm{S}$ or its derivatives via copolymerization occurred rather inefficiently. In contrast, better results were achieved from method 2 involving samples derived from FluO-TEMPO. The ATR-FTIR spectra of the graft copolymer display the presence of the following characteristic bands of grafted S: (i) the signals at 699 and $756 \mathrm{~cm}^{-1}$ assigned to the out-of-plane bending of the $\mathrm{CH}$ groups of the PS aromatic ring, ${ }^{34}$ (ii) the band at $1493 \mathrm{~cm}^{-1}$ attributed to the stretching vibrations of the carbons in the PS aromatic ring, (iii) the signal 
centered at ca. $1600 \mathrm{~cm}^{-1}$ related to the aromatic $\mathrm{C}=\mathrm{C}$ stretching bond in PS, and finally (iv) the stretching of $=\mathrm{CH}$ aromatic, as well as the overtone of the ring between 2000 and $1665 \mathrm{~cm}^{-1}$ were highlighted suggesting even a good covering of films surface (Fig. 3).

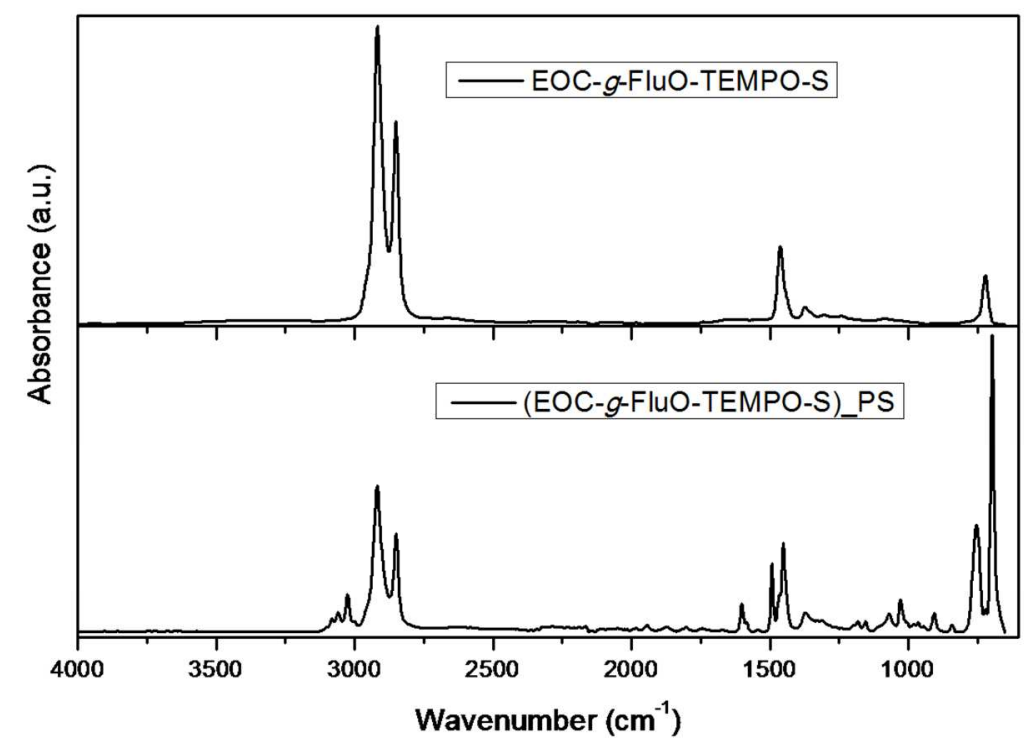

Figure 3 ATR-FTIR spectra of EOC-g-FluO-TEMPO-S (upper spectrum) and (EOC-g-FluOTEMPO-S)_PS (lower spectrum, run 7, Table 1).

The FTIR spectrum (Fig. S3) of the graft copolymer achieved from method $2 b$ that used bulk functionalized FluO-TEMPO polyolefin is very similar to that of Fig. 3, in which the signals assigned to the PS grafts were all clearly visible (Fig. S3). Furthermore, the successful insertion of PS grafts onto the polyolefin backbone was also confirmed by NMR spectroscopy. After purification with acetone, ${ }^{1} \mathrm{H}-\mathrm{NMR}$ spectrum of the purified and dried graft copolymer clearly shows the presence of the signals in the range $6.0-7.5 \mathrm{ppm}$ attributed to the aromatic ring (Fig. 4). The resolved small signal at about 3.75 (t) ppm was attributed to $-\mathrm{CH}$ in $\alpha$-position to oxygen of the nitroxide (see inset in the Fig. 4), confirming the NMP reaction. The PS content in this sample was estimated to be $65 \mathrm{~mol} \%$ of the copolymer. 


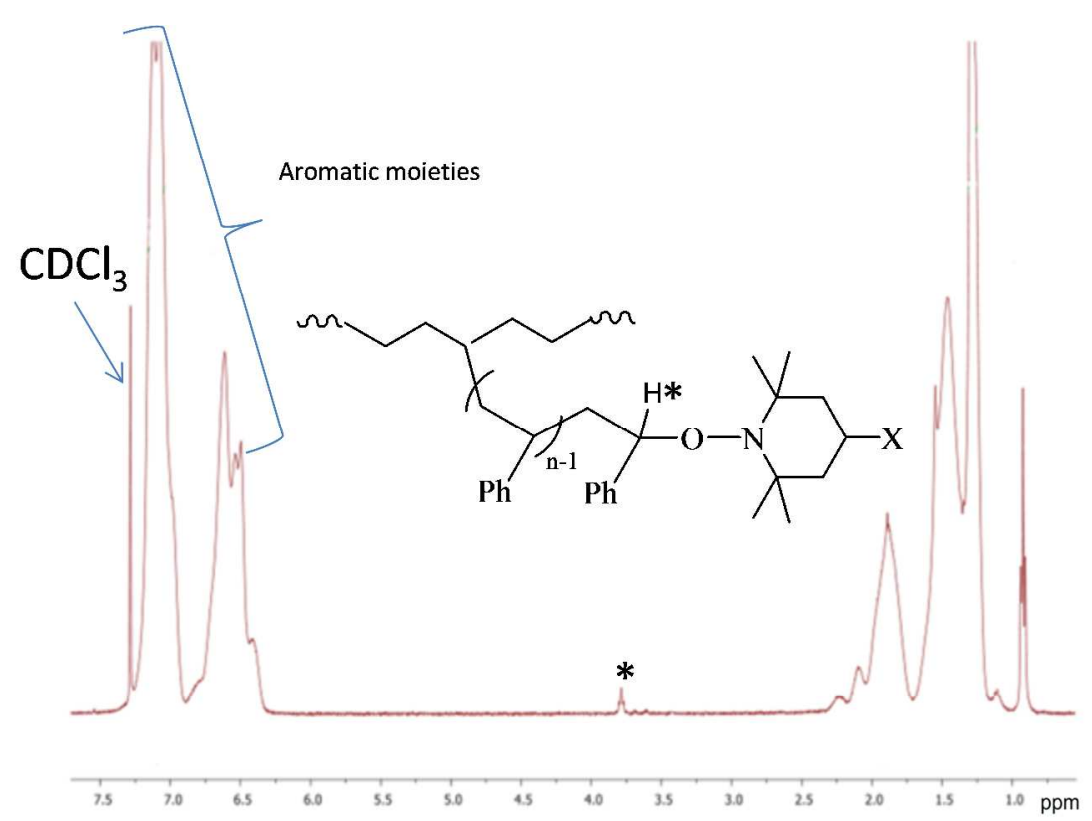

Figure $4{ }^{1} \mathrm{H}-\mathrm{NMR}$ spectrum of (EOC-g-FluO-TEMPO)_PS (run 8 Table 1; recorded in $\mathrm{CDCl}_{3}$ ).

The grafting of PS from FluO-TEMPO was also evidenced from solid state ${ }^{19} \mathrm{~F}-\mathrm{NMR}$ spectroscopy. The spectrum of (EOC-g-FluO-TEMPO-S)_PS (Fig. 5) displays two characteristic signals centered at ca. -81 and $-115 \mathrm{ppm}$, assigned to $-\mathrm{CF}_{3}$ and $-\left(\mathrm{CF}_{2}\right)_{5}$ of the fluorinated alkyl chain of FluO-TEMPO, respectively. By assuming that the NMP is characterized by the reversible coupling reaction between the alkyl(macro)radical and the nitroxide to form the dormant species, the presence of the FluO-TEMPO, likely linked to the grafted PS chains, suggested the growth of the chains by NMP reaction, confirming the hypothesis about the grafting mechanism. 


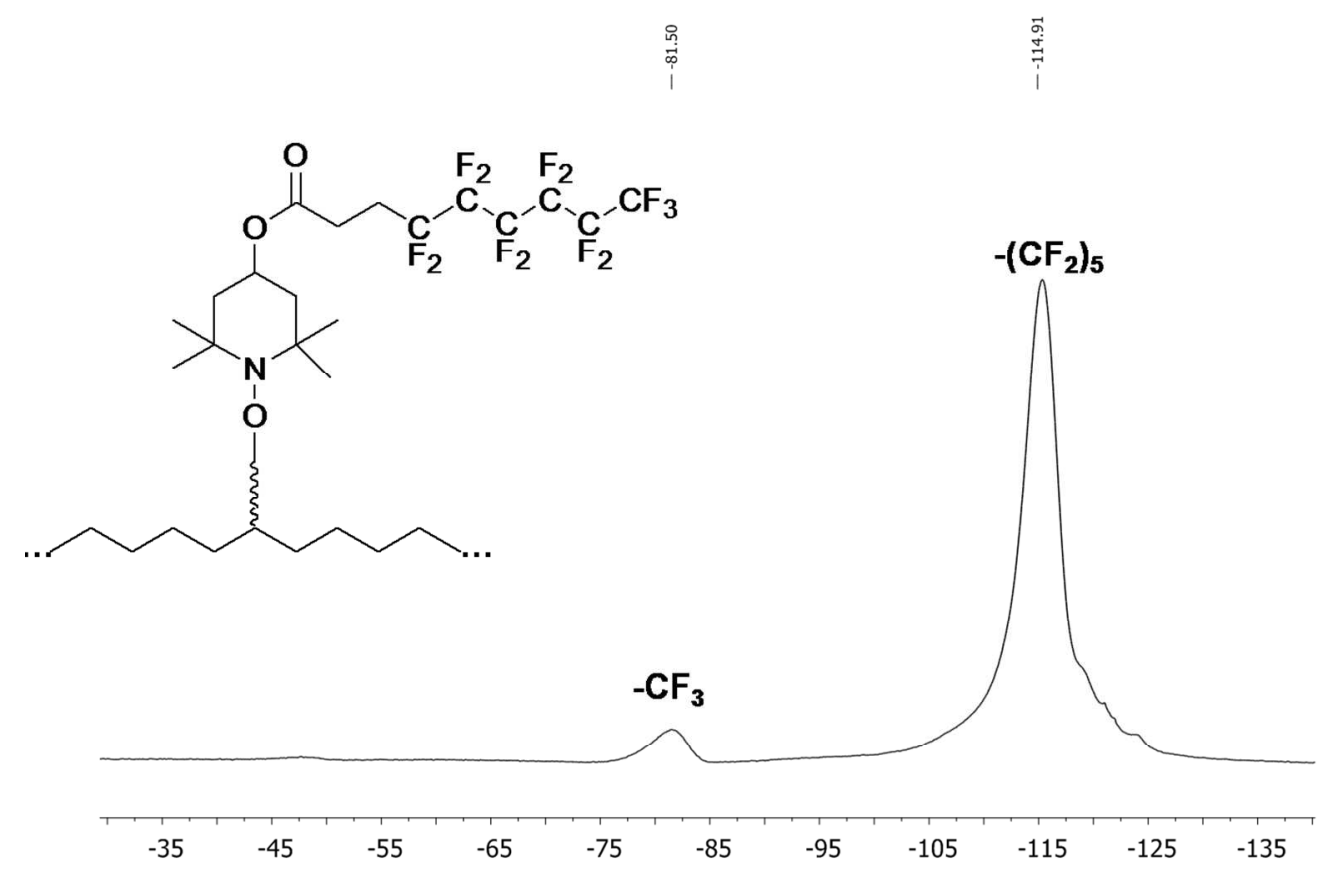

Figure 5: Solid state ${ }^{19}$ F-NMR spectrum of the (EOC-g-FluO-TEMPO)-S_PS graft copolymer (run 7 Table 1).

Figure 6 exhibits the SEC traces of both the EOC-g-FluO-TEMPO macroinitiator and the (EOC-g-FluO-TEMPO)_PS graft copolymer, recorded by using RI detector. Though the molar mass-conversion linear relationship was not established, the copolymer displayed a higher average molar mass, $M_{\mathrm{n}},(165000 \mathrm{~g} / \mathrm{mol})$ and a lower polydispersity $\left(M_{\mathrm{w}} / M_{\mathrm{n}}=1.46\right)$ with respect to that of the macroinitiator $\left(M_{\mathrm{n}}=77000 \mathrm{~g} / \mathrm{mol}, M_{\mathrm{w}} / M_{\mathrm{n}}=1.84\right)$. In any case, the monomodal shape of this chromatogram indicates the absence of any residual homopolymer and the complete initiation of the macroinitiator during the NMP process. Moreover, the SEC trace of the (EOC-gFluO-TEMPO)_PS copolymer recorded with a UV detector $(\lambda=254 \mathrm{~nm})$ was much more intense than that of the corresponding macroinitiator EOC-g-FluO-TEMPO (Fig. S4), hence confirming the actual incorporation of the polystyrene grafts in the polymer structure. 


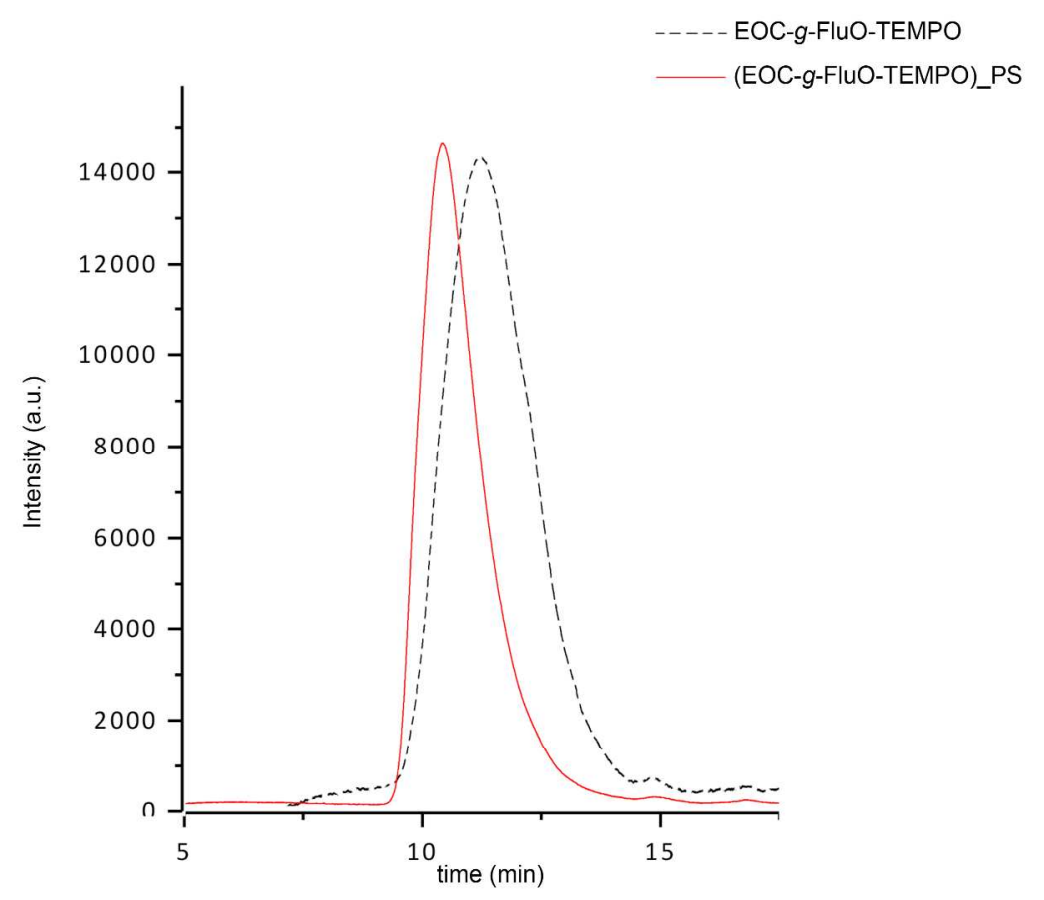

Figure 6 SEC traces (RI detector) of macroinitiator EOC-g-FluO-TEMPO and graft copolymer (EOC-g-FluO-TEMPO)_PS(run 8, Table 1).

For comparison, by considering that the polymerizations were carried out in bulk, a blank experiment involving the non-functionalized polymer, EOC, and S was carried out to definitely assess the role of grafted TEMPO derivative as the macroinitiator. Run 9 in Table 1 (for the preparation of EOC_PS) performed by using the same experimental conditions (EOC/S ratio, time and temperature) of the collected (EOC-g-FluO-TEMPO-S)_PS sample proved that only in presence of TEMPO grafted moiety, the growing of PS chains can be achieved. In fact, the IR spectrum of the collected sample (EOC_PS, run 9, Table 1) showed only the absorption bands attributed to EOC and no frequencies assigned to PS grafts were observed (Fig. S5).

The apparently low grafting yield (less than $10 \%$ ) for the samples derived from method 1 could be due to the use of solvent, which slowed down the polymerization rate and significantly decreases the monomer conversion of TEMPO mediated radical polymerization systems. ${ }^{11}$ 
Moreover, in these runs (runs 1-3, Table 1), the monomer amount was significantly smaller than that used in method $2\left([\mathrm{~S}]_{\mathrm{o}} /[\mathrm{X}-\mathrm{TEMPO}]_{\mathrm{o}}=180 \mathrm{vs}[\mathrm{S}]_{\mathrm{o}} /[\text { FluO-TEMPO }]_{\mathrm{o}}=6900\right.$ or 7100$)$.

Furthermore, the thermal stability of grafted alkoxyamine is a key parameter that plays a fundamental role in these copolymerization reactions. It was shown ${ }^{29}$ that the homolytic cleavage of alkoxyammine $\mathrm{R}_{1} \mathrm{R}_{2} \mathrm{R}_{3} \mathrm{C}-\mathrm{ON}<$ bond (generating the macroradical and the free nitroxide), necessary to initiate the copolymerization reaction, occurred at relatively low temperature for the moieties derived from FluO-TEMPO grafting. ${ }^{30}$ This is, indeed, closer to the temperature of $\mathrm{S}$ copolymerization employed for the grafting from reactions, justifying the higher yield for EOC$g$-FluO-TEMPO and EOC-g-FluO-TEMPO-S samples. Conversely, the high thermal stability of grafted AzO-TEMPO and THiO-TEMPO derivatives ${ }^{29,28}$ limited the amount of macroradicals necessary to start the copolymerization of S. This led to lower polymer grafting.

\section{Thermal properties of the graft copolymers}

The thermal properties of the graft copolymers were studied by thermogravimetric analysis (TGA) and differential scanning calorimetry (DSC). The TGA thermograms of the copolymers obtained from method 1 (Fig. S6-S7) compared to those of the polyethylene starting material and of the PS sample obtained by free radical polymerization used as reference $\left(M_{\mathrm{n}}=42000 \mathrm{~g} / \mathrm{mol}\right.$ and $\left.M_{\mathrm{w}} / M_{\mathrm{n}}=3.56\right)$, evidenced that the degradation of the grafted copolymers occurred at lower temperature $^{35,36}$ although close to that of the starting polyolefins. This may be attributed to the bond cleavages between the functionalized polyolefin and the PS grafts. However, the degradation profiles are really similar and, as evidenced by derivative of thermogravimetric (DTG) plots, only one step can be detected (Fig. S8-S9). In these conditions, it seems that the degradation of the aliphatic polymer backbone in the copolymerized samples is poorly affected by the degradation of the aromatic grafts, in agreement with the low PS grafting occurrence, and 
the weight loss due to the styrene moiety cannot be distinguished. ${ }^{35}$
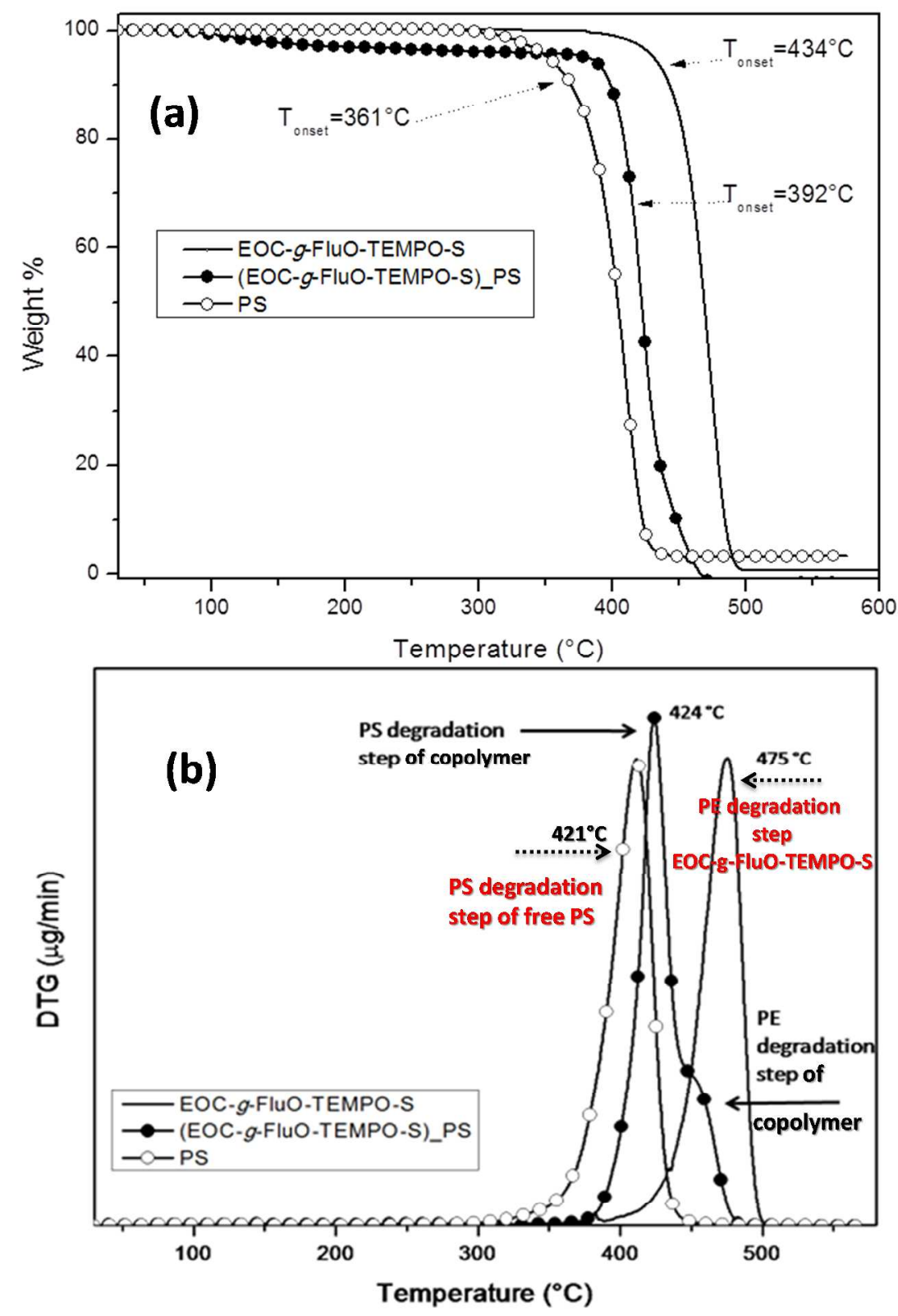

Figure 7: (a) TGA thermograms of (EOC-g-FluO-TEMPO-S)_PS and the EOC-g-FluOTEMPO-S (Run 7 in Table 1). The analysis was carried out under nitrogen flow; (b) Derivative thermogravimetric plots (under nitrogen) of EOC-g-FluO-TEMPO-S, (EOC-g-FluO-TEMPOS)_PS and PS (Run7 in Table 1). 
Instead, the copolymers obtained by method 2, by using EOC grafted with FluO-TEMPO as macroinitiators, showed significant difference in terms of thermal stability with respect to their corresponding starting samples. As for example, the TGA profile of copolymer (EOC-gFluO-TEMPO-S)_PS evidenced a shift in the degradation temperature (as onset of the main degradation step, $\mathrm{T}_{\text {onset }}$ ) of about $40^{\circ} \mathrm{C}$ toward lower temperature with respect to the value of starting EOC-g-FluO-TEMPO-S (Fig 7a) and moving close to the $\mathrm{T}_{\text {onset }}$ value characteristic of PS $\left(361^{\circ} \mathrm{C}\right)$. In addition, although the TGA thermogram suggested a behavior resembling a onestep degradation path, for (EOC-g-FluO-TEMPO-S)_PS graft copolymer, the DTG evidenced a bimodal peak indicating the presence of copolymer fractions having thermal stability depending on their composition (Fig 7b). A main peak really close to that of the DTG characteristic of free PS (used as reference) and a shoulder at higher temperature, partially overlapped to the peak assigned to pristine EOC-g-FluO-TEMPO-S were observed. The first one was attributable to PS grafts in the copolymer, while the latter to polyethylene backbone, confirming the occurrence of grafting from reaction in good yield (this sample contains $65 \mathrm{~mol} \%$ of PS grafts).

The DSC analysis of the same copolymer (EOC-g-FluO-TEMPO-S)_PS is reported in Figure 8 in comparison with that of pristine EOC-g-FluO-TEMPO-S. Both samples showed the $\operatorname{Tg}$ (at ca. $-45^{\circ} \mathrm{C}$ ) and the large melting point of pristine functionalized $\mathrm{EOC}^{34}$ (Fig. 8). In addition, the DSC curve of (EOC-g-FluO-TEMPO-S)_PS displayed the clear presence of a second order transition in the 90 to $110^{\circ} \mathrm{C}$ range. This new transition can be assigned to the $T_{g}$ of PS chains ${ }^{37}$ grafted to EOC owing to FluO-TEMPO moieties as even proved by DSC analysis of PS used as the reference evidencing a glass transition in the same temperature range. The small difference between the two values $T_{g(\mathrm{PS})}=100{ }^{\circ} \mathrm{C}$ and $T_{g(\mathrm{PS} \text { grafts of copolymer })}=94{ }^{\circ} \mathrm{C}$ is presumably due to different PS chain lengths, lower in the case of (EOC-g-FluO-TEMPO-S)_PS 
copolymer. ${ }^{37}$ Similar results were also obtained for (EOC-g-FluO-TEMPO)_PS graft copolymer which displayed a $T_{g}$ value of the PS segments in the same temperature range (Fig. S10). This observation further evidences the successful PS grafting from the polyolefin matrix.

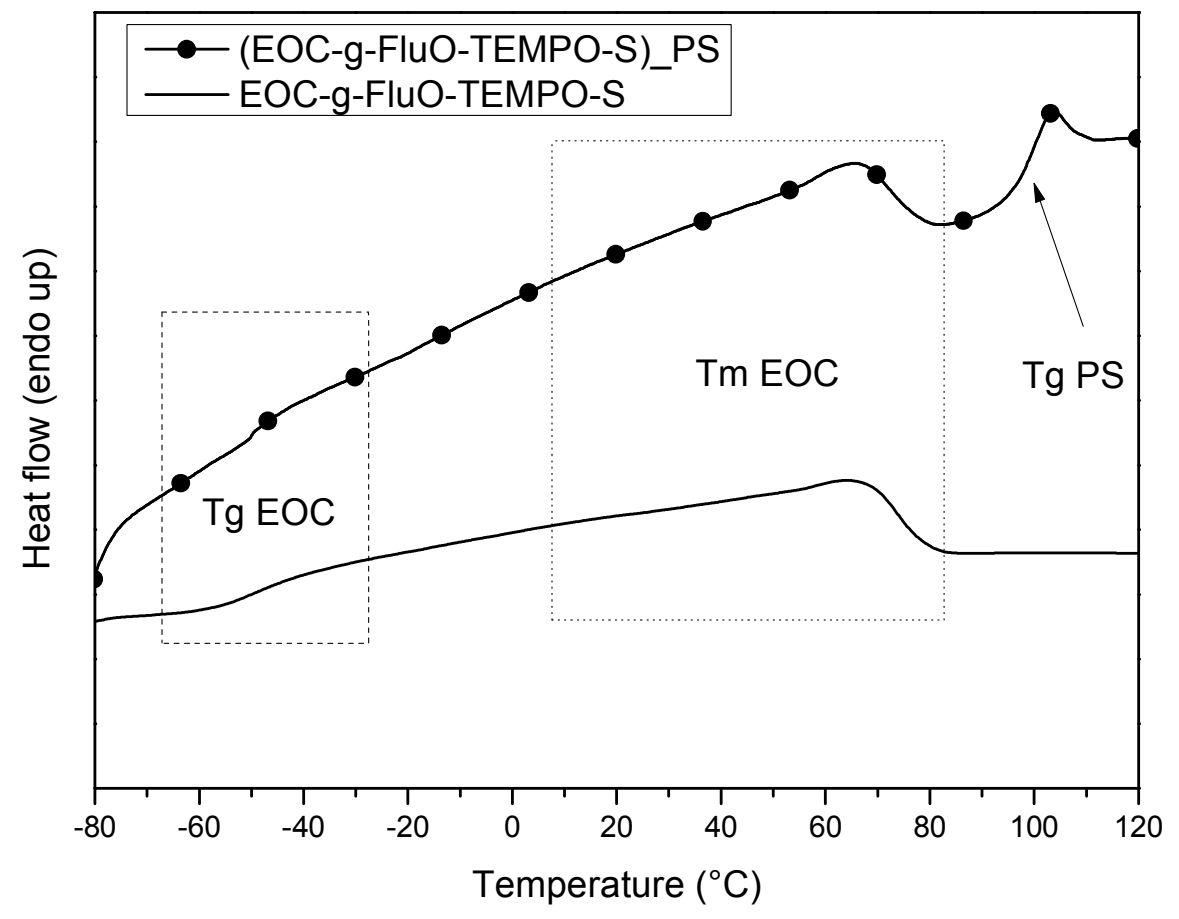

Figure 8: DSC thermograms of EOC-g-FluO-TEMPO-S and (EOC-g-FluO-TEMPO-S)_PS (Run 7 in Table 1).

\section{Conclusions}

Polyolefin alkoxyamines, obtained through the NRC reaction of AzO-TEMPO, THiO-TEMPO and FluO-TEMPO with polyolefin macroradicals, were used as macroinitiators for the synthesis of polyolefin-g-polystyrene graft copolymers via "grafting from" technique using styrene or styrene derivatives as (co)monomers. ATR-FTIR, NMR, TGA, and DSC analyses enabled to fully highlight the grafting occurrence; in particular IR spectra of copolymers exhibited the frequencies attributed to aromatic moieties and the TGA thermograms evidenced a lower 
degradation temperature for the grafted copolymers compared to those of polyolefin starting materials. This behavior was caused by the cleavage of bonds between the polyolefin and the PS grafts. The collected results evidenced that the grafting yield was significantly higher when the macro-alkoxyamine bearing FluO-TEMPO was used as starting polyolefin. The grafting reaction from polyolefins functionalized with AzO-TEMPO and THiO-TEMPO was carried out with lower amount of comonomer and in presence of solvent which caused a reduction in the polymerization rate and significantly limited the monomer conversion. In addition, the same alkoxyamines showed higher thermal stability than EOC-g-FluO-TEMPO by reducing the availability and the amount of species able to initiate the styrene polymerization. Consistently, the highest grafting efficiency was obtained by using the more reactive fluorine-based alkoxyamine EOC-g-FluO-TEMPO combined with the use of the largest $[\mathrm{S}]_{\mathrm{o}} /[\mathrm{TEMPO}]_{\mathrm{o}}$ ratio. These conditions ensured a PS grafted content of $65 \mathrm{~mol} \%$ based on copolymer composition, as evidenced by ${ }^{1}$ H-NMR spectroscopy. These results pointed out that the functional polyolefinalkoxyamines can be considered as "activated macromolecules" to be exploited as macroinitiators for the subsequent preparation of graft polymers with tuned/tailored structureproperties. Further monomers (such as methacrylates, dienes, halogenated alkenes) may also be attempted to prepare novel graft copolymers.

\section{Corresponding Author}

*E-mail: bruno.ameduri@enscm.fr (B.A.).

*E-mail: elisa.passaglia@pi.iccom.cnr.it (E.P.)

\section{Acknowledgements}


I.D. thanks EU for providing a Mobility Grant for Higher Education Learning Agreement for traineeships, Erasmus + HE-2015. S.B. thanks the French National Agency for support (ANR grant FLUPOL, No. ANR-14-CE07-0012). A special thanks to Dr. Francesca Cicogna and Dr. Serena Coiai for their hard work in contributing to the discussion of the results, as well as Prof. G. Silly who recorded the solid state NMR spectra. Arkema is also acknowledged for supplying Lacqtene ${ }^{\circledR}$ while B.A. thanks the French Fluorine Network (GIS) for support.

Electronic Supplementary Information (ESI) available: Additional analyses including NMR and IR spectra, and DSC thermograms of the synthesized (co)polymers.

\section{Notes and references}

1. N. K. Boaen and M. A. Hillmyer, Chem. Soc. Rev, , 2005, 34, 267-275.

2. L. S. Boffa and B. M. Novak, Chem. Rev., 2000, 100, 1479-1494.

3. C. Feng, Y. Li, D. Yang, J. Hu, X. Zhang and X. Huang, Chem. Soc. Rev, 2011, 40, 1282-1295.

4. G. Moad, Prog. Polym. Sci., 1999, 24, 81-142.

5. K. E. Russell, Prog. Polym. Sci., 2002, 27, 1007-1038.

6. B. Kluperman, Kluperman; Introduction to reversible deactivation radical polymerization, in N.K. Singha; J.W. Mays, (eds) pp 1-15; Smithers Rapra 2017, Shrewsbury.

7. J. Nicolas, Y. Guillaneuf, C. Lefay, D. Bertin, D. Gigmes and B. Charleux, Prog. Polym. Sci., 2013, 38, 63-235. 
8. M. K. Georges, R. P. N. Veregin, P. M. Kazmaier and G. K. Hamer, Macromolecules, 1993, 26, 2987-2988.

9. C. J. Hawker, A. W. Bosman and E. Harth, Chem. Rev., 2001, 101, 3661-3688.

10. M. Mariani, M. Lelli, K. Sparnacci and M. Laus, J. Polym. Sci. A: Polym. Chem., 1999, 37, $1237-1244$.

11. A. Narumi, T. Matsuda, H. Kaga, T. Satoh and T. Kakuchi, Polymer, 2002, 43, 48354840.

12. J. Ding, C. Chuy and S. Holdcroft, Adv. Funct. Mater., 2002, 12, 389-394.

13. B. Boutevin, Y. Hervaud, A. Boulahna and M. El Asri, Macromolecules, 2002, 35, 65116516.

14. D. Hua, W. Deng, J. Tang, J. Cheng and X. Zhu, Int. J. Biol. Macromol., 2008, 43, 43-47.

15. A. Debuigne, R. Poli, C. Jerome, R. Jerome and C. Detrembleur, Prog. Polym. Sci., 2009, 34, 211-239.

16. Nitroxide Mediated Polymerization: From Fundamentals toApplications in Materials Science; Gigmes, D., Ed.; RSC: Oxford, 2015

17. M. Abbasian, H. Namazi and A. A. Entezami, Polym. Adv. Technol., 2004, 15, 606-611.

18. S. Mohajery, S. Rahmani and A. A. Entezami, Polym. Adv. Technol., 2008, 19, 15281535.

19. M. Abbasian, M. Shahparian and S. E. S. Bonab, Iran. Polym. J., 2013, 22, 209-218.

20. F. Cicogna, S. Coiai, E. Passaglia, I. Tucci, L. Ricci, F. Ciardelli and A. Batistini, J. Polym. Sci. A: Polym. Chem., 2011, 49, 781-795.

21. F. Cicogna, S. Coiai, C. Pinzino, F. Ciardelli and E. Passaglia, React. Funct. Polym., 2012, 72, 695-702. 
22. J. Bonilla-Cruz, E. Saldívar-Guerra, J. R. Torres-Lubián, R. Guerrero-Santos, B. LópezCarpy and G. Luna-Bárcenas, Macromol. Chem. Phys., 2008, 209, 2268-2283.

23. E. Saldivar-Guerra, J. Bonilla-Cruz, B. Hernandez-Mireles and G. Ramirez-Manzanares, Macromol. Symp., 2009, 283-284, 110-119.

24. Y. Higaki, H. Otsuka, T. Endo and A. Takahara, Macromolecules, 2003, 36, 1494-1499.

25. S. Hietala, S. Holmberg, M. Karjalainen, J. Nasman, M. Paronen, R. Serimaa, F. Sundholm and S. Vahvaselka, J. Mater. Chem., 1997, 7, 721-726.

26. S. Hietala, M. Koel, E. Skou, M. Elomaa and F. Sundholm, J. Mater. Chem., 1998, 8, 1127-1132.

27. M. Elomaa, S. Hietala, M. Paronen, N. Walsby, K. Jokela, R. Serimaa, M. Torkkeli, T. Lehtinen, G. Sundholm and F. Sundholm, J. Mater. Chem., 2000, 10, 2678-2684.

28. M. Paronen, F. Sundholm, D. Ostrovskii, P. Jacobsson, G. Jeschke, E. Rauhala and P. Tikkanen, Chem. Mater., 2003, 15, 4447-4455.

29. F. Cicogna, I. Domenichelli, S. Coiai, F. Bellina, M. Messi, R. Spiniello and E. Passaglia, Polymer, 2016, 82, 366-377.

30. I. Domenichelli, S. Coiai, C. Pinzino, S. Taddei, E. Martinelli and F. Cicogna, Eur. Polym. J., 2017, 87, 24-38.

31. F. Cicogna, I. Domenichelli, S. Coiai, F. Bellina, M. Lessi, R. Spiniello and E. Passaglia, Polymer, 2016, 82, 366-377.

32. F. Liu, B.-K. Zhu and Y.-Y. Xu, Appl. Surf. Sci., 2006, 253, 2096-2101.

33. G. Zorn, J. E. Baio, T. Weidner, V. Migonney and D. G. Castner, Langmuir, 2011, 27, 13104-13112. 
34. F. Meng, Q. Zhang, G. Wang, H. Wang and Y. Gao, J. App. Polym. Sci., 2015, 132, 41671.

35. T. A. Sherazi, S. Ahmad, M. A. Kashmiri, D. S. Kim and M. D. Guiver, J. Membr. Sci., 2009, 333, 59-67.

36. T. A. Sherazi, T. Rehman, S. A. R. Naqvi, A. J. Shaikh, S. A. Shahzad, G. Abbas, R. Raza and A. Waseem, Appl. Surf. Sci., 2015, 359, 593-560.

37. T. C. Chung, H. L. Lu and R. D. Ding, Macromolecules, 1997, 30, 1272-1278. 
Table of contents entry

Styrene and substituted styrene grafted functional polyolefins via nitroxide mediated polymerization

Ilaria Domenichelli, Sanjib Banerjee, Sara Taddei, Elisa Martinelli, Elisa Passaglia and Bruno Ameduri

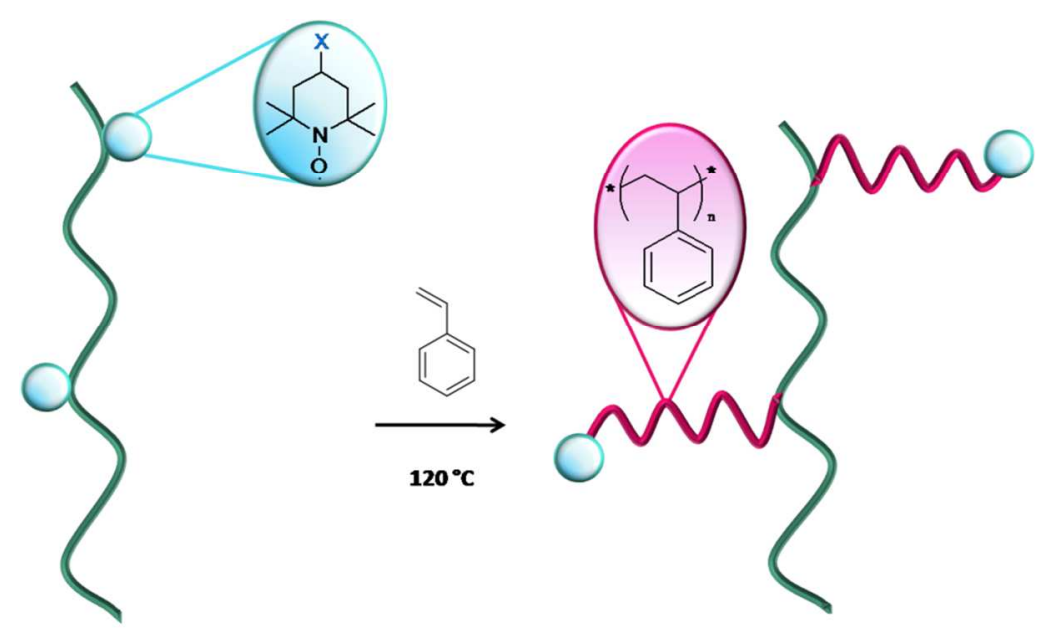


Functionalized polyolefins bearing alkoxyamines derived from nitroxide radical coupling reaction were successfully used as the macroinitiator to graft polystyrene or substituted polystyrene brushes via nitroxide mediated polymerization. 


\section{Electronic Supplementary Information}

Styrene and substituted styrene grafted functional polyolefins via nitroxide mediated polymerization

Ilaria Domenichelli, $t^{\mathrm{a}, \mathrm{b}}$ Sanjib Banerjee, $\dagger^{\mathrm{c}, \#}$ Sara Taddei, ${ }^{\mathrm{d}}$ Elisa Martinelli, ${ }^{\mathrm{d}}$ Elisa Passaglia ${ }^{\mathrm{b}}$ and Bruno Ameduri ${ }^{\mathrm{c}, *}$

${ }^{a}$ Scuola Normale Superiore, Piazza dei Cavalieri 7, 56126 Pisa, Italy

${ }^{\mathrm{b}}$ CNR-ICCOM UOS Pisa, Via Moruzzi 1, 56124 Pisa, Italy

${ }^{\mathrm{c}}$ Ingénierie et Architectures Macromoléculaires, Institut Charles Gerhardt, UMR 5253 CNRS, UM, ENSCM, Place Eugène Bataillon, 34095 Montpellier Cedex 5, France

${ }^{\mathrm{d}}$ Dipartimento di Chimica e Chimica Industriale, Via Moruzzi 13, 56124 Pisa, Italy

\#Present address: Material Science and Technology Division, CSIR-National Institute for Interdisciplinary Science and Technology, Thiruvananthapuram 695019, Kerala, India

† These authors contributed equally to this manuscript.

*Corresponding Author: E-mail: bruno.ameduri@enscm.fr (B.A.) elisa.passaglia@pi.iccom.cnr.it (E.P.) 
A

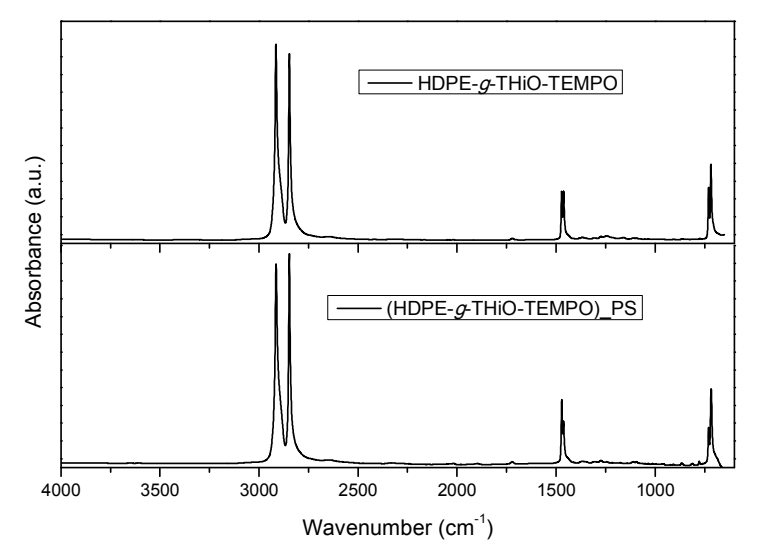

C

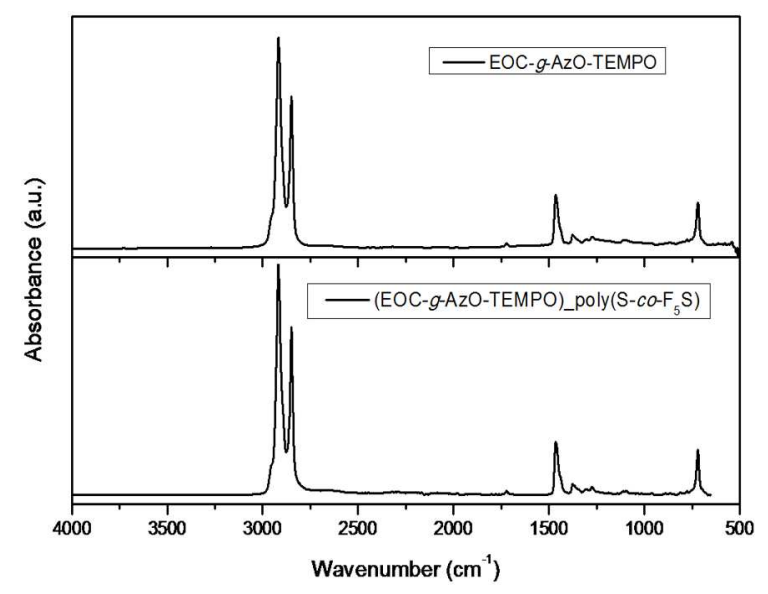

B

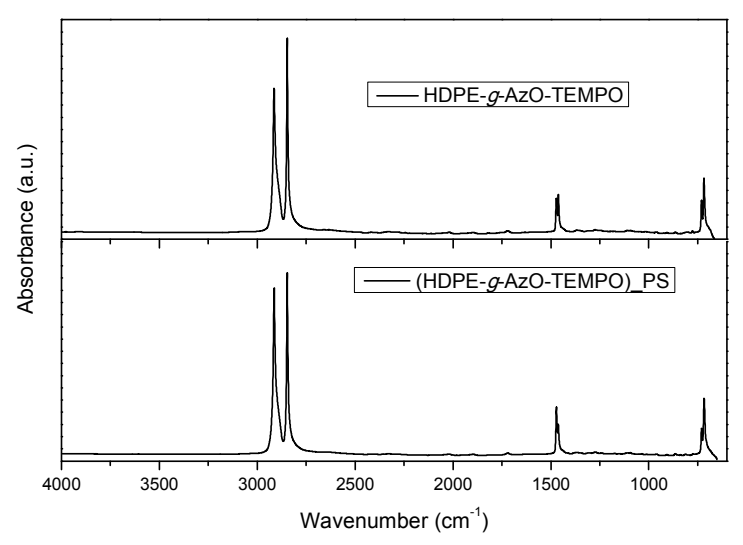

D

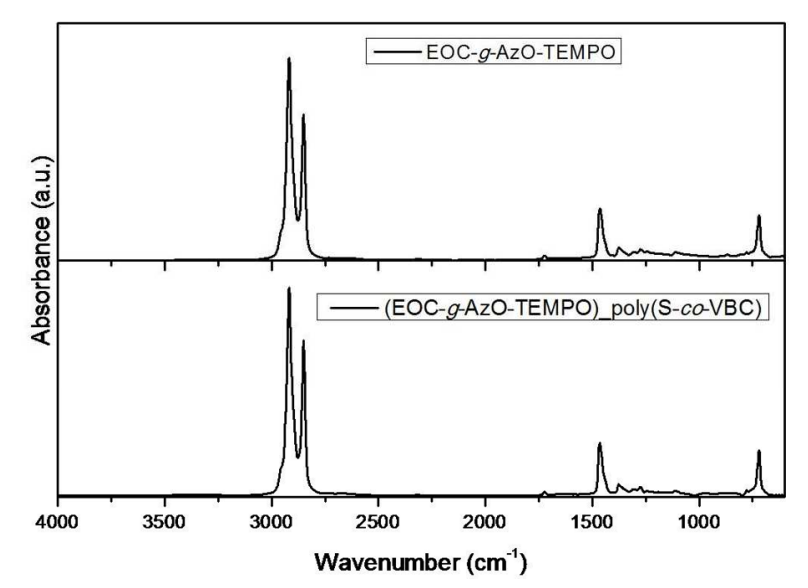

Fig. S1 ATR-FTIR spectra of A: HDPE-g-THiO-TEMPO, (HDPE-g-THiO-TEMPO)_PS, B: HDPE-g-AzO-TEMPO, (HDPE-g-AzO-TEMPO)_PS, C: EOC-g-AzO-TEMPO, (EOC-g-AzOTEMPO)_poly(S-co-F 5 S) and D: EOC-g-AzO-TEMPO, (EOC-g-AzO-TEMPO)_poly(S-coVBC) (runs 2-5 Table 1). 


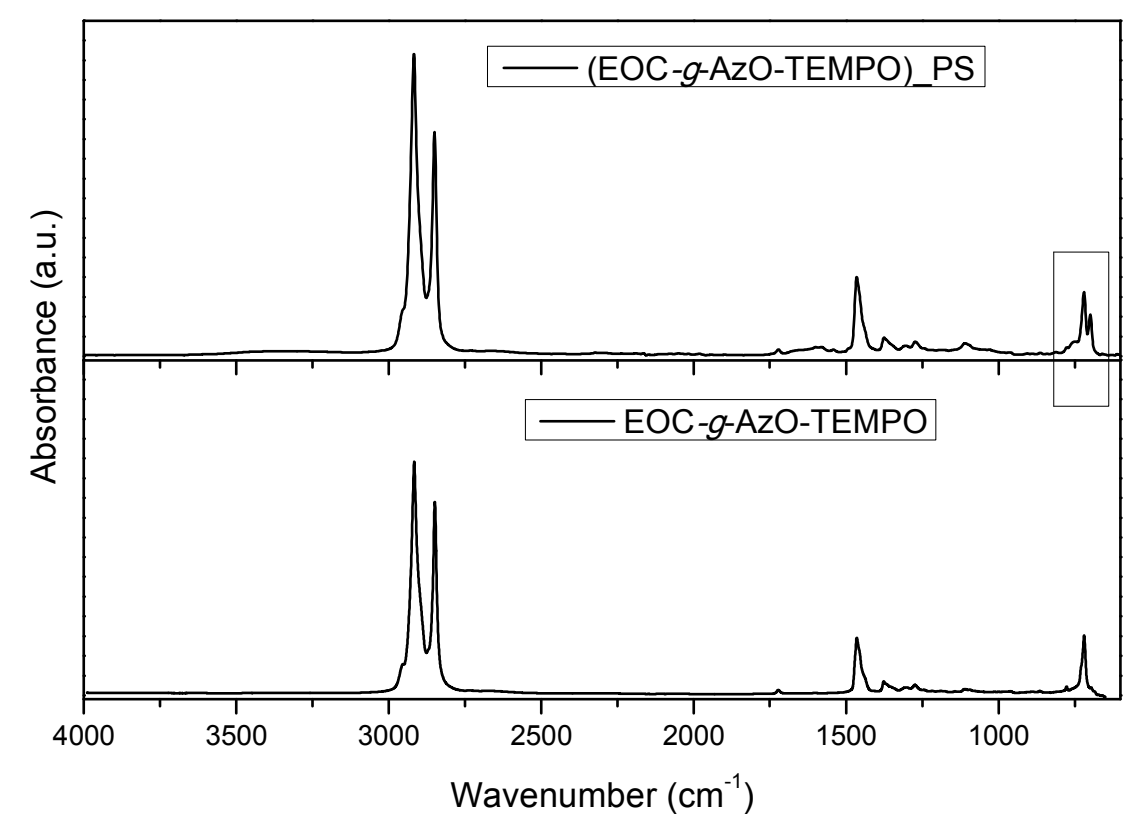

Fig. S2 ATR-FTIR spectra of the EOC-g-AzO-TEMPO and (EOC-g-AzO-TEMPO)_PS (run 1, Table 1) 


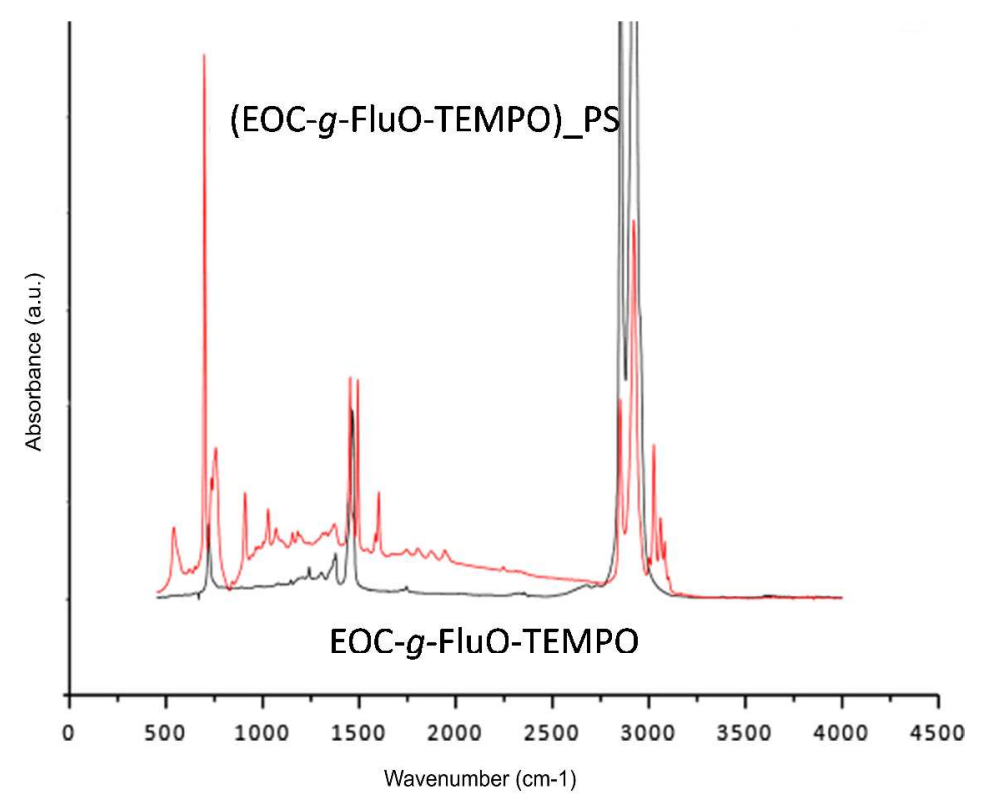

Fig. S3 FT-IR spectra of EOC-g-FluO-TEMPO (black curve) and (EOC-g-FluO-TEMPO)_PS (red curve). (run 8 Table 1) 


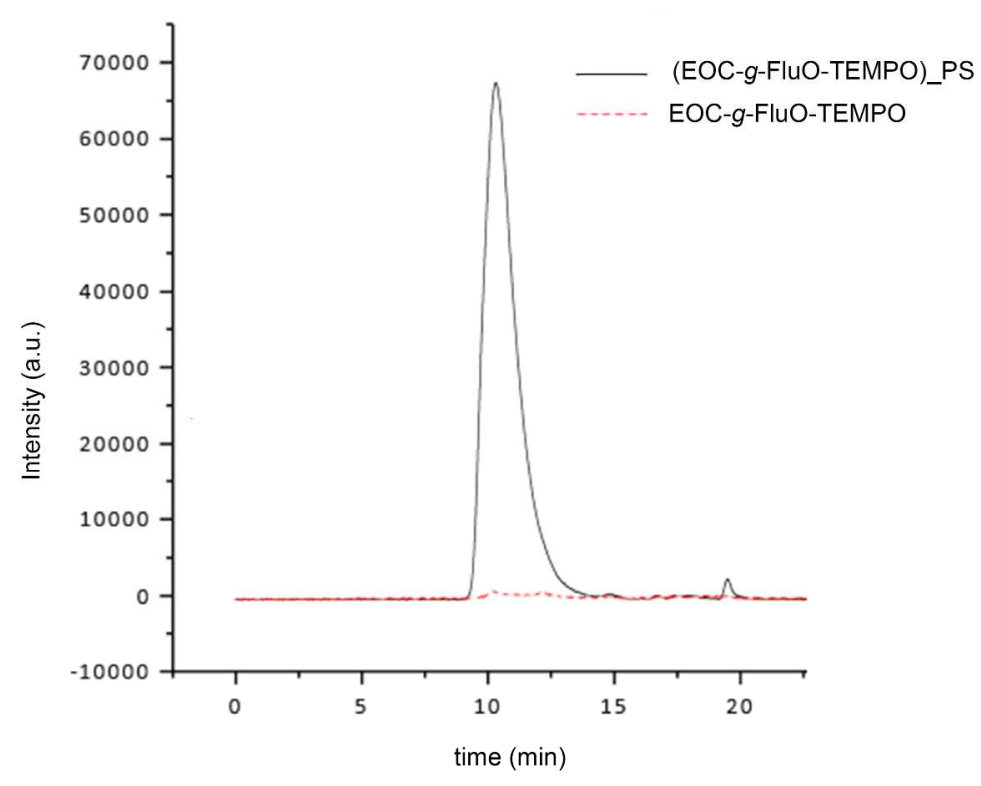

Fig. S4 SEC traces (UV detector, $\lambda=254 \mathrm{~nm}$ ) of macroinitiator EOC- $g$-FluO-TEMPO(dotted red line) and graft copolymer (EOC-g-FluO-TEMPO)_PS (black line). (run 8 Table 1) 


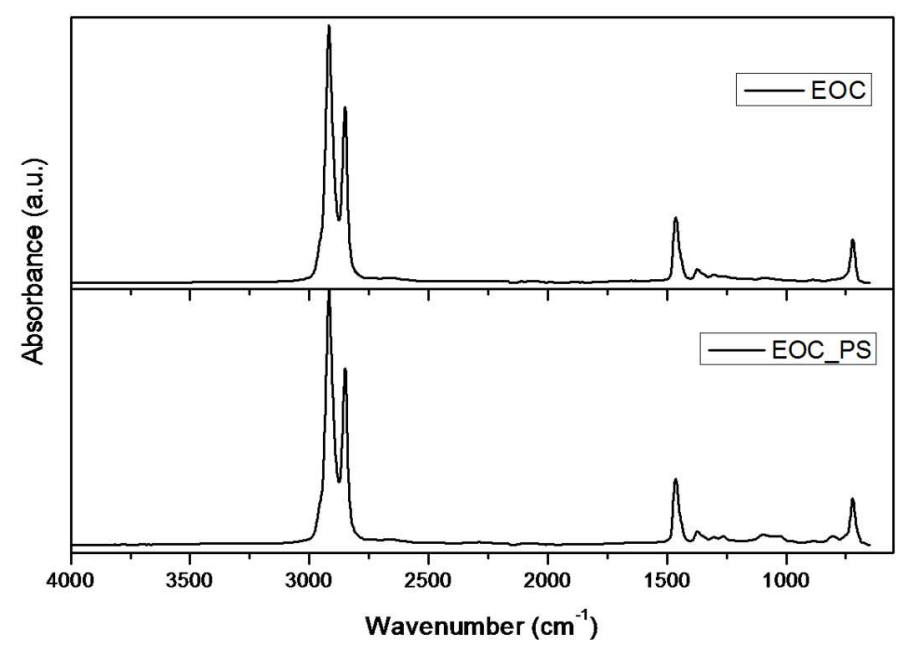

Fig. S5 ATR-FTIR spectra of EOC sample and EOC_PS sample corresponding to EOC treated with $\mathrm{S}$ in the bulk (run 9 Table 1) 

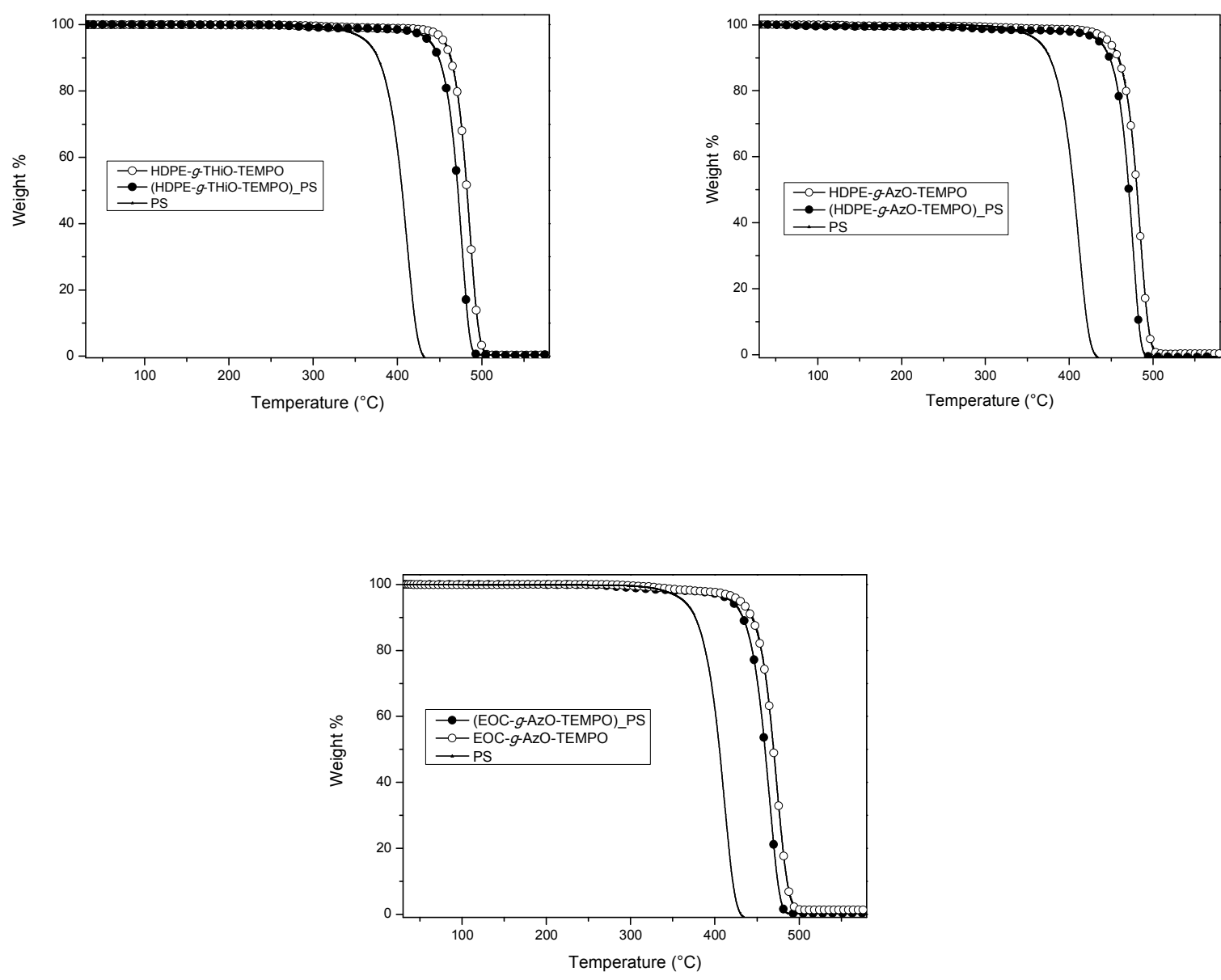

Fig. S6 TGA thermograms of the functionalized HDPE and EOC samples before and after the treatment with styrene and the free PS (runs 1-3 Table 1).The analysis was carried out under nitrogen flow. 

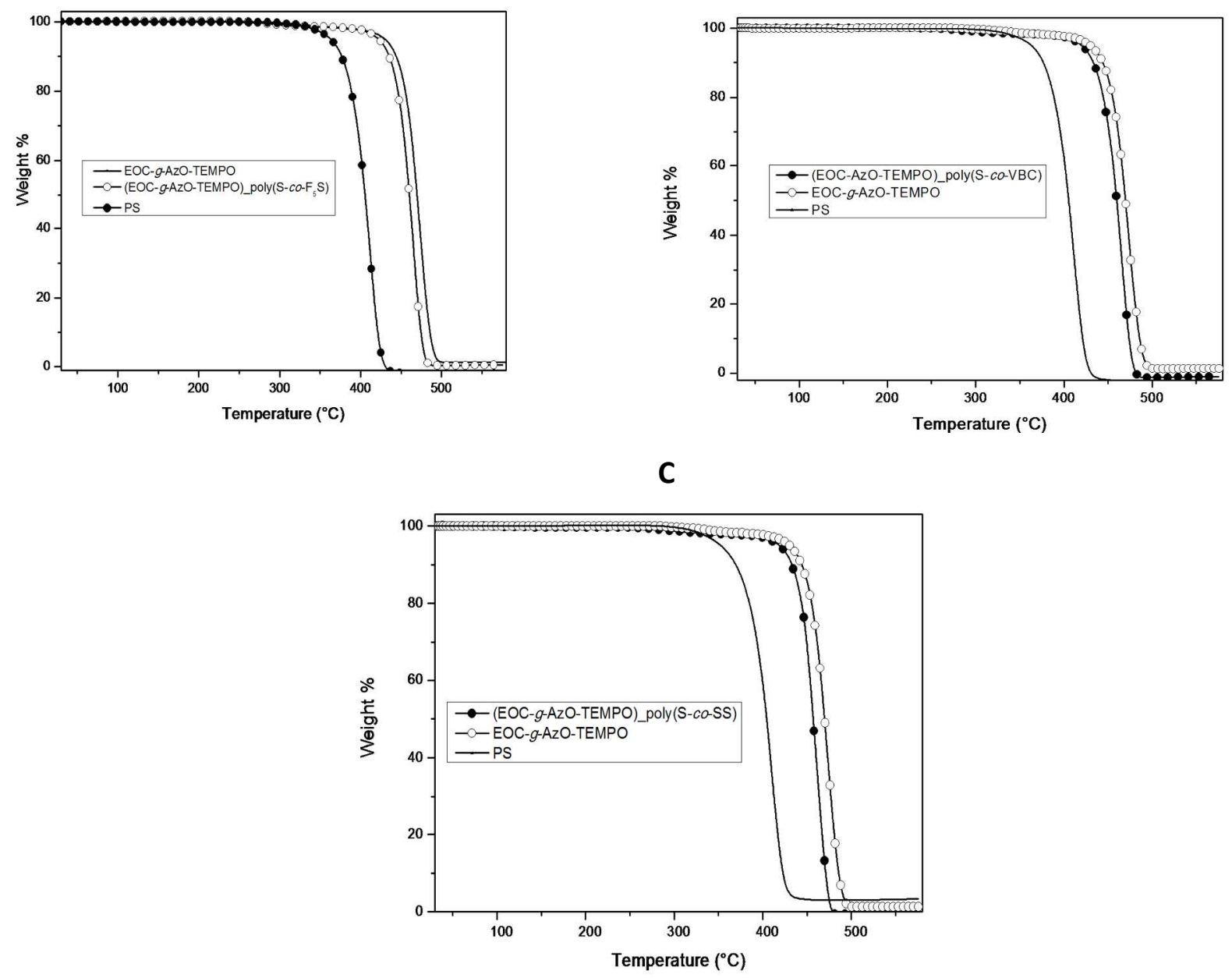

Fig. S7 TGA thermograms of A: EOC-g-AzO-TEMPO, (EOC-g-AzO-TEMPO)_poly(S-co-F 5 S) and pristine PS, B: EOC-g-AzO-TEMPO, (EOC-g-AzO-TEMPO)_poly(S-co-VBC)and pristine PS and C: EOC-g-AzO-TEMPO, (EOC-g-AzO-TEMPO)_poly(S-co-SS) and pristine PS (runs 46 Table 1). These analyses were carried out under nitrogen flow. 
A

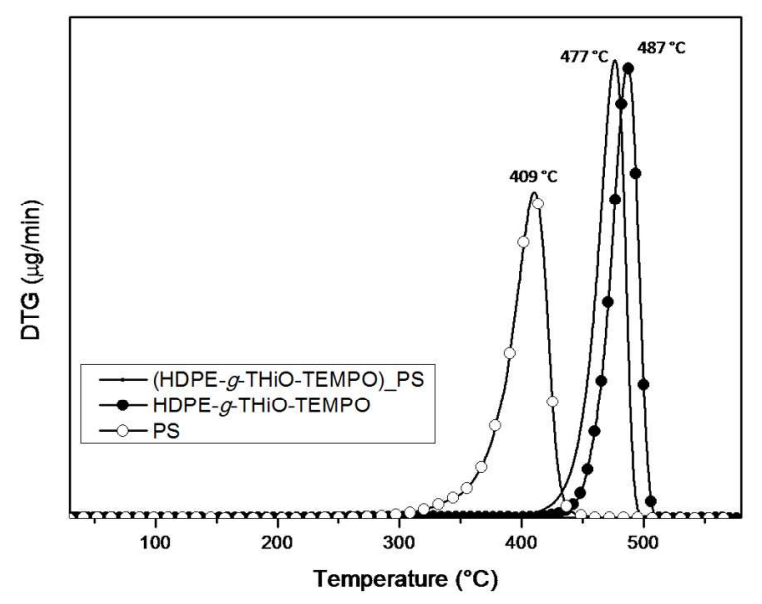

B

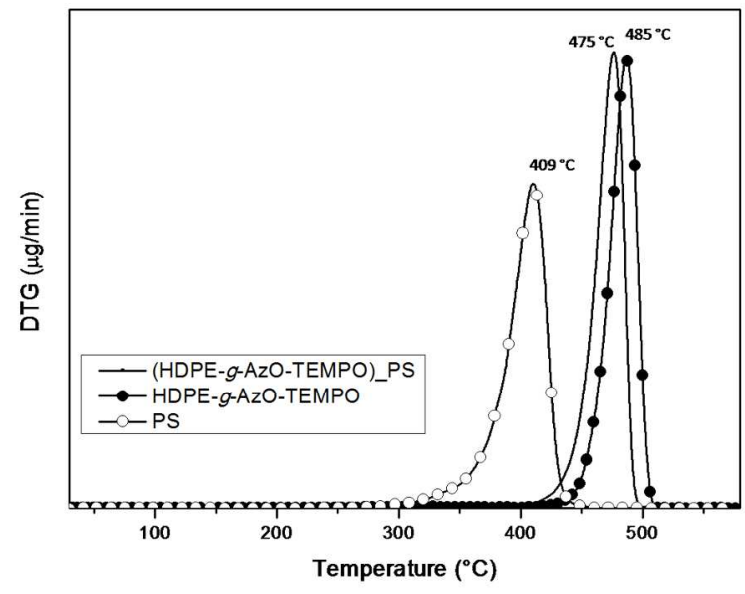

C

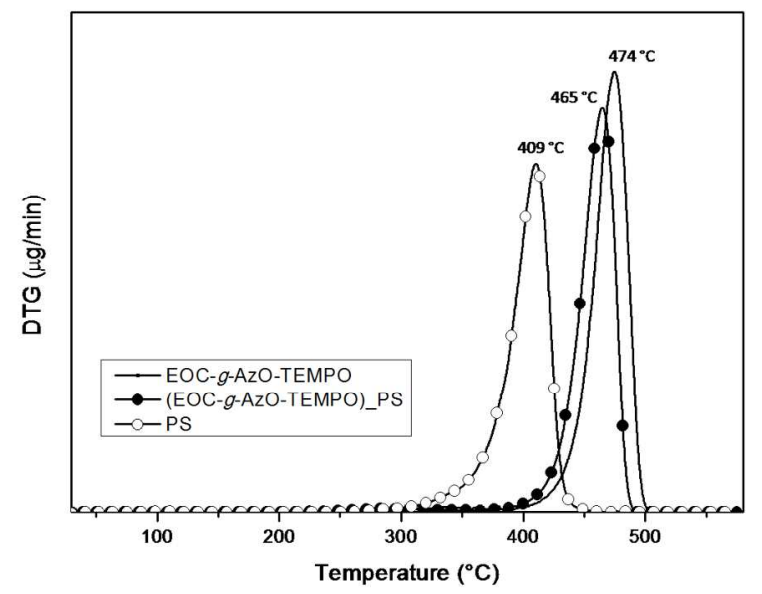

Fig. S8 Derivative of thermogravimetric (DTG) plots of: A: HDPE-g-ThiO-TEMPO, (HDPE-gThiO-TEMPO)_PSand free PS, B: HDPE-g-AzO-TEMPO, (HDPE-g-AzO-TEMPO)_PSand free PS, C: EOC-g-AzO-TEMPO, (EOC-g-AzO-TEMPO)_PS and free PS (runs 1-3 Table 1). These analyses were carried out under nitrogen flow. 
A

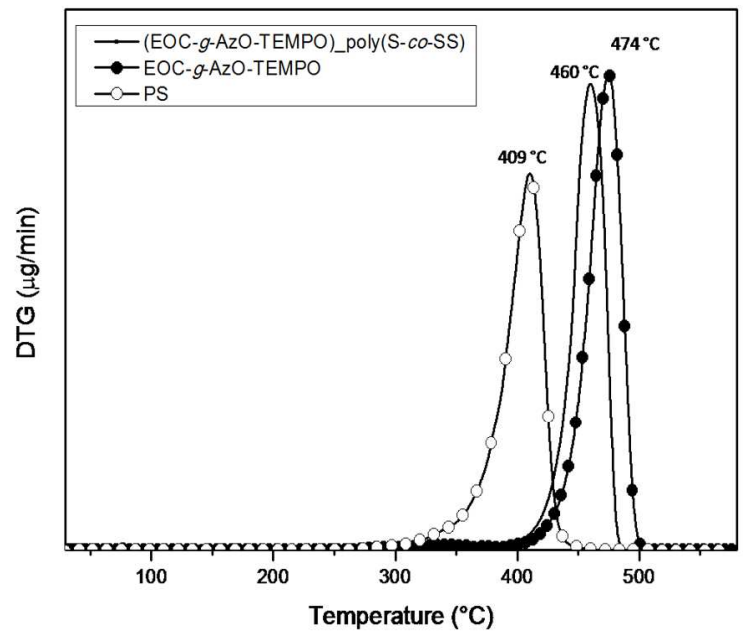

B

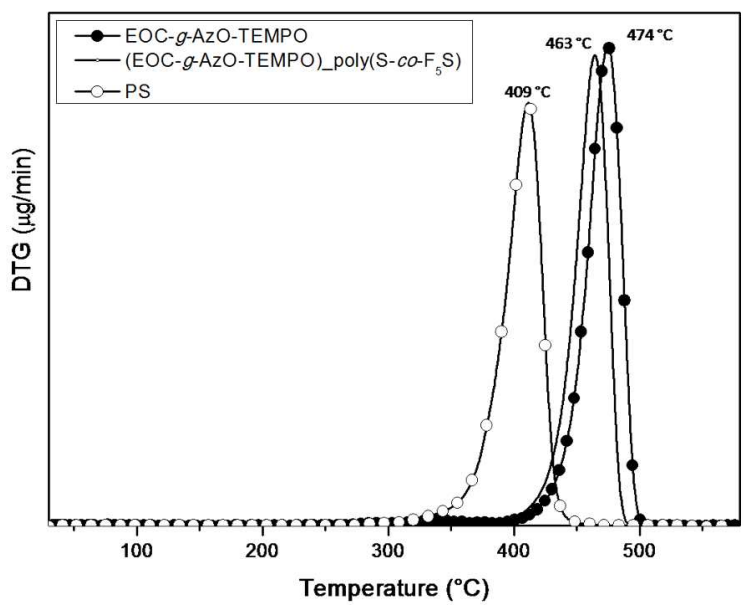

C

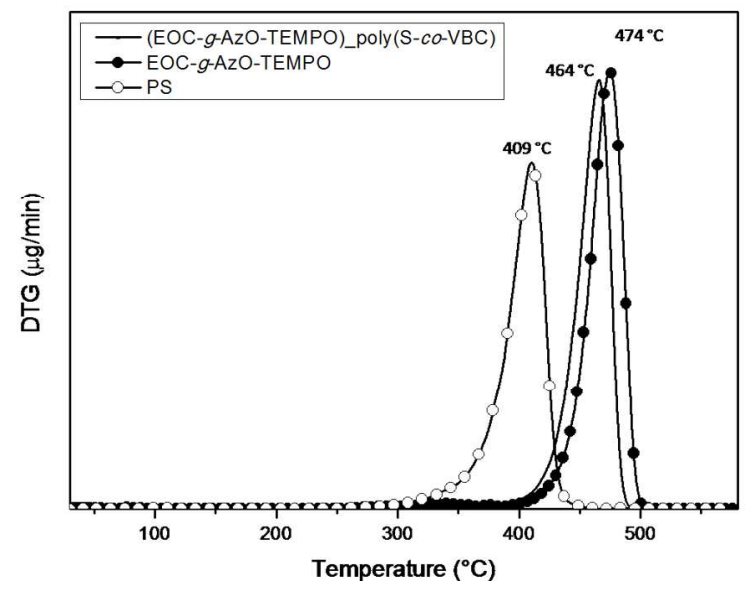

Fig. S9 Derivative of thermogravimetric (DTG) plots A: EOC-g-AzO-TEMPO, (EOC-g-AzOTEMPO)_poly(S-co-SS) and pristine PS, B: EOC-g-AzO-TEMPO, (EOC-g-AzOTEMPO)_poly(S-co- $\left.\mathrm{F}_{5} \mathrm{~S}\right)$ and pristine PS and C: EOC-g-AzO-TEMPO, (EOC-g-AzOTEMPO)_poly(S-co-VBC)and pristine PS. (runs 4-6 Table 1). These analyses were carried out under nitrogen flow. 


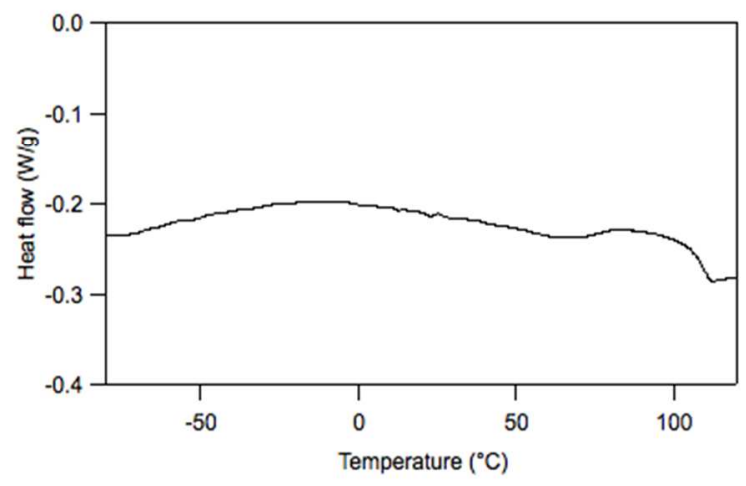

Fig. S10. DSC thermograms of (EOC-g-FluO-TEMPO)_PS (run 8 Table 1) 